\title{
Nonadiabatic orientation, toroidal current, and induced magnetic field in $\mathrm{BeO}$ molecules
}

\author{
Ingo Barth, ${ }^{1, a)}$ Luis Serrano-Andrés, ${ }^{2}$ and Tamar Seideman ${ }^{3}$ \\ ${ }^{1}$ Institut für Chemie und Biochemie, Freie Universität Berlin, Takustr. 3, 14195 Berlin, Germany \\ ${ }^{2}$ Instituto de Ciencia Molecular, Universitat de València, Apartado 22085, ES-46071 Valencia, Spain \\ ${ }^{3}$ Department of Chemistry, Northwestern University, 2145 Sheridan Road, \\ Evanston, Illinois 60208-3113, USA
}

(Received 10 July 2008; accepted 15 September 2008; published online 23 October 2008)

\begin{abstract}
It is predicted that oriented $\mathrm{BeO}$ molecules would give rise to unprecedentedly strong, unidirectional electric ring current and an associated magnetic field upon excitation by a right or left circularly polarized laser pulse into the first excited degenerate singlet state. The strong toroidal electric ring current of this state is dominated by the ring current of the $1 \pi_{ \pm}$orbital about the molecular axis. Our predictions are based on the analysis of the orbital composition of the states involved and are substantiated by high level electronic structure calculations and wavepacket simulations of the laser-driven orientation and excitation dynamics. (0) 2008 American Institute of Physics.
\end{abstract}

[DOI: $10.1063 / 1.2994737$ ]

\section{INTRODUCTION}

With the rapid advance of attosecond physics, ${ }^{1-3}$ it becomes relevant to explore numerically the dynamical motion of electrons within molecular systems. On the subfemtosecond time scale, the vibrational modes are frozen, introducing an opportunity to trigger and directly probe electronic dynamics, ${ }^{4-13}$ and coupled electron-nuclear dynamics. ${ }^{14-17}$

An interesting example of electron dynamics in bound states of neutral molecules is unidirectional electron circulation, or electric ring currents, and associated magnetic fields, occurring in degenerate excited states that carry nonzero electronic angular momentum. Such currents have been numerically observed in atoms and ions, ${ }^{18}$ in oriented linear molecules, ${ }^{19,20}$ and in oriented aromatic molecules. ${ }^{21-25}$ Electric ring currents and associated magnetic fields can also be generated in two-dimensional nanosized quantum rings by means of picosecond laser pulses, e.g., by two shaped timedelayed laser pulses with perpendicular linear polarizations, ${ }^{26}$ by circularly polarized laser pulses,${ }^{27}$ or by optimized laser pulses designed through optimal-control theory. ${ }^{28}$ Electron circulation in chiral aromatic molecules can be manipulated by means of linearly polarized laser pulses ${ }^{29,30}$ but the direction of the electric ring currents alternates periodically after the end of laser pulses due to the nondegeneracy of the excited states. Finally, in an analogous way, circularly polarized laser pulses can also induce unidirectional nuclear pseudorotation about the molecular axis of linear triatomic molecules such as ${ }^{114} \mathrm{CdH}_{2}$ (Ref. 31) or $\mathrm{FHF}^{-} .{ }^{32}$ It has been proposed that high harmonic generation by means of elliptically polarized laser pulses could serve to observe this phenomenon in the laboratory. ${ }^{33}$ In general, one expects the ring currents and induced magnetic fields to be

\footnotetext{
${ }^{a)}$ Author to whom correspondence should be addressed. Electronic mail: barth@chemie.fu-berlin.de.
}

stronger for diatomic molecules and atoms or ions than for aromatic molecules because the former correspond to much smaller current radii than the latter. ${ }^{18,19}$

While electronic ring currents are a very general and well-understood effect, their properties depend crucially on the degenerate electronic states that carry them. Before laboratory realization could be considered, it is thus important to first develop guidelines for the design (or choice) of a molecule that would maximize the effect. Given the sensitivity of the ring currents to the details of the electronic structure, it is relevant also to make quantitative predictions of the observables based on high level electronic structure calculations. Finally, a major limitation of the approach is its reliance on orientation of the molecule with respect to the exciting laser polarization axes. Orientation is crucial here since although states with nonzero electronic angular momentum (e.g., $\pi_{ \pm}$that depend as $e^{ \pm i \phi}$ on the angle of rotation about the body-fixed $Z$-axis $\phi$ ) are associated with unidirectional electron circulation in the molecular frame, an isotropic mixture of these states does not exhibit unidirectional current in the laboratory frame. The requirement of alignment is common to all spectroscopies that attempt to image the electronic structure or dynamics, including the method of orbital tomography via high harmonic generation $^{34}$ and time-resolved photoelectron angular distributions. ${ }^{35}$ The additional requirement of orientation is specific to the present approach and results from the handedness (right versus left) of the observable considered. It need be remarked that in the laboratory it is much more challenging to induce orientation than to induce alignment. ${ }^{36,37}$ All previous studies of ring currents have assumed that the molecule is perfectly oriented with respect to the excitation laser field. It is thus important to first assess the degree of orientation that can in principle be induced in a molecule that is expected to support strong ring currents and explore the experimental requirements to that end.

The goal of the present work is thus threefold. We first 
detail the set of criteria that candidate molecules should satisfy and explain our choice of the $\mathrm{BeO}$ molecule as a suitable model that is anticipated to support much higher currents than those computed before. Next we carry out high level electronic structure calculations of the ground and singlet excited states of $\mathrm{BeO}$. We proceed with quantum calculations of orientation of $\mathrm{BeO}$ by moderately intense laser pulses (see Refs. 36 and 37 for an early and a recent review, respectively). Finally, we report calculations of the ring current and associated magnetic field in $\mathrm{BeO}$.

The $\mathrm{BeO}$ molecule has long attracted the attention of $a b$ initio quantum chemistry because of its large dipole moment, a magnitude particularly difficult to compute accurately in polar diatomic systems. Earlier configuration interaction (CI), ${ }^{38,39}$ coupled-cluster $\mathrm{CCSD}+\mathrm{T}(\mathrm{CCSD}),{ }^{40}$ and $\operatorname{CCSD}(\mathrm{T})$ (Ref. 41) studies focused in the ground state $X^{1} \Sigma^{+}$, whereas the first excited state $A^{1} \Pi$ was computed at low CI levels. ${ }^{42}$ Only recently were the excited states of the molecule reported at the MRD-CI level of calculation ${ }^{43}$ (very recently also for the $\mathrm{BeO}^{+}$cation, see Ref. 44). Here, we will carry out multiconfigurational perturbative second-order computations employing the CASPT2 method $^{45}$ to analyze the properties of the ground and singlet excited states of the molecule.

In the following sections, we show that the strongest unidirectional electric ring current and induced magnetic field in the first excited degenerate bound singlet state of a neutral molecule are achieved for the $A{ }^{1} \Pi_{+}$state of the oriented $\mathrm{BeO}$ molecule. Our arguments for choosing the $\mathrm{BeO}$ molecule are detailed in Sec. II, where we describe also our CASPT2 results. We present quantum simulations of the orientation of the $\mathrm{BeO}$ molecule by means of a linearly polarized picosecond laser pulse in Sec. III and of the excitation of the strong electric ring current and induced magnetic field in the oriented $\mathrm{BeO}$ molecule in Sec. IV, see also Fig. 1. The final section provides a brief summary and conclusions.

\section{MODEL SYSTEM}

We open this section by discussing the criteria for choice of a suitable molecule that would maximize the electric ring current. We look for a class of molecules with following set of properties: (i) the molecule is electric neutral; (ii) the ground singlet state of the molecule is bound and nondegenerate, carrying no electric ring current, for example, a ${ }^{1} \Sigma,{ }^{1} A$, or ${ }^{1} B$ state; (iii) an excited degenerate singlet state, for example, a ${ }^{1} \Pi_{ \pm}$or ${ }^{1} E_{ \pm}$state, is energetically located below the ion states, and hence could be excited with no risk of multiphoton ionizations; and (iv) the excited degenerate state is bound, and therefore the electric ring current and induced magnetic field can persist in the excited bound state for a long period (where "long" is quantified below).

Within the theory of electric ring currents and induced magnetic fields, it was predicted that in one-electron ions the effects are strongest for atomic $2 p_{ \pm}$orbitals and for large nuclear charges $Z$, i.e., the electric ring current and induced magnetic field at the nucleus increase with the nuclear charge $Z$ as $Z^{2}$ and $Z^{3}$, respectively. ${ }^{18}$ For many-electron systems $Z$ can be approximately replaced by the effective nuclear

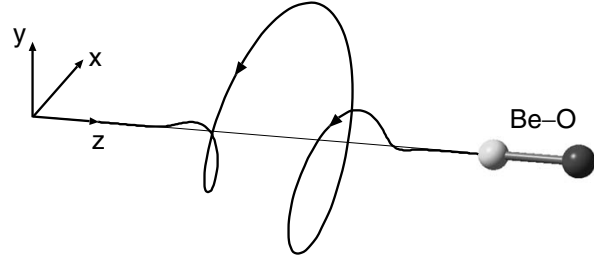

FIG. 1. Optimized right circularly polarized, ultrashort few-cycle laser pulse incident on the oriented $\mathrm{BeO}$ molecule and propagating along the molecular and space-fixed $z$-axis during the revival of the field-free rotational wavepacket. Field-free orientation of the $\mathrm{BeO}$ molecule is induced by means of a picosecond laser pulse that is linearly polarized along the excitation field propagation direction axis (not shown). The parameters of the circularly polarized laser pulse are $\mathcal{E}_{c}=10.05 \mathrm{GV} \mathrm{m}^{-1}\left(I_{\max , c}=26.8 \mathrm{TW} \mathrm{cm}^{-2}\right), \hbar \omega_{c}$ $=1.04 \mathrm{eV}=8388.2 \mathrm{hc} \mathrm{cm} \mathrm{cm}^{-1}$ (with a laser cycle of duration $\Delta t_{c}=3.98 \mathrm{fs}$ ), $\tau_{c}=2.74 \mathrm{fs}\left(t_{p, c}=21.2 \mathrm{fs}\right)$. The arrows indicate the sequence of few laser cycles as observed in the molecular frame when the pulse passes by.

charge $Z_{\text {eff }}$, which can be estimated using the Slater rules. ${ }^{46,47}$ For molecules, the linear combination of atomic orbitals molecular orbital (LCAO-MO) approximation can be used to estimate the ring currents and associated magnetic fields based on our understanding of the atomic case. For example, the predicted electric ring current about the $\mathrm{Al}$ nucleus and the induced magnetic field at the $\mathrm{Al}$ nucleus in the $A{ }^{1} \Pi_{ \pm}$ state of the $\mathrm{AlCl}$ molecule are $\left|I_{\mathrm{Al}}\right| \approx 430 \mu \mathrm{A}$ and $\left|\mathbf{B}\left(\mathbf{r}_{\mathrm{Al}}\right)\right|$ $\approx 6.0 \mathrm{~T}$, where the LCAO-MO expansion of the lowest unoccupied molecular orbital (LUMO) $4 \pi_{ \pm}$was used. ${ }^{19}$ These estimates compare well with the CASSCF values $\left|I_{\mathrm{Al}}\right|$ $=313 \mu \mathrm{A}$ and $\left|\mathbf{B}\left(\mathbf{r}_{\mathrm{Al}}\right)\right|=7.7 \mathrm{~T} .{ }^{19}$ Furthermore, smaller current radii yield stronger electric ring currents and induced magnetic fields. This result can be readily understood within the current loop model. Thus, the effects are stronger in linear molecules, where the current radii are small, than in ringshaped molecules, where they are typically larger. For example, the electric ring current about the $\mathrm{Mg}$ nucleus and induced magnetic field at the $\mathrm{Mg}$ nucleus in the $4{ }^{1} E_{u \pm}$ state of the ring-shaped molecule $\mathrm{Mg}$-porphyrin are $|I|$ $=84.5 \mu \mathrm{A}$ and $\left|\mathbf{B}\left(\mathbf{r}_{\mathrm{Mg}}\right)\right|=0.16 \mathrm{~T}$, respectively. The much weaker effects as compared to those computed for $\mathrm{AlCl}$ are predominantly the result of the different current radii in the two cases, $R=6.32 a_{0}$ for Mg-porphyrin whereas $R=0.18 a_{0}$ for $\mathrm{AlCl}{ }^{19,22}$

In the atomic case, strong effects are associated with $2 p_{ \pm}$orbitals, and hence the effects in the $1 \pi_{ \pm}$orbitals of diatomic molecules are expected to be strong, as these are dominated by the $2 p_{ \pm}$orbitals of the atomic constituents. For polyatomic linear molecules (with three or more atoms), one expects the ring currents and induced magnetic fields to be smaller than for diatomics because the molecular orbitals have contributions of more atomic orbitals and therefore the dominant atomic $2 p_{ \pm}$orbitals usually have smaller weights than in diatomic molecules. Many of the diatomic molecules consisting of atoms with larger $Z_{\text {eff }}$, for example, Na, $\mathrm{Mg}$, and $\mathrm{Al}$, are ruled out because the $1 \pi_{ \pm}$orbitals lie energetically sufficiently low that the first ionization energy is smaller than the $\Pi_{ \pm}$state excitation energy. Diatomic molecules containing $\mathrm{F}$ or $\mathrm{Ne}$ atoms do not satisfy all conditions (i)-(iv), for example, the first excited states $A{ }^{1} \Pi_{ \pm}$of $\mathrm{HeNe}, \mathrm{HF}$, and $\mathrm{LiF}$ molecules are dissociative and those of $\mathrm{BeF}$ and $\mathrm{BF}$ do not correspond to the orbital transitions 


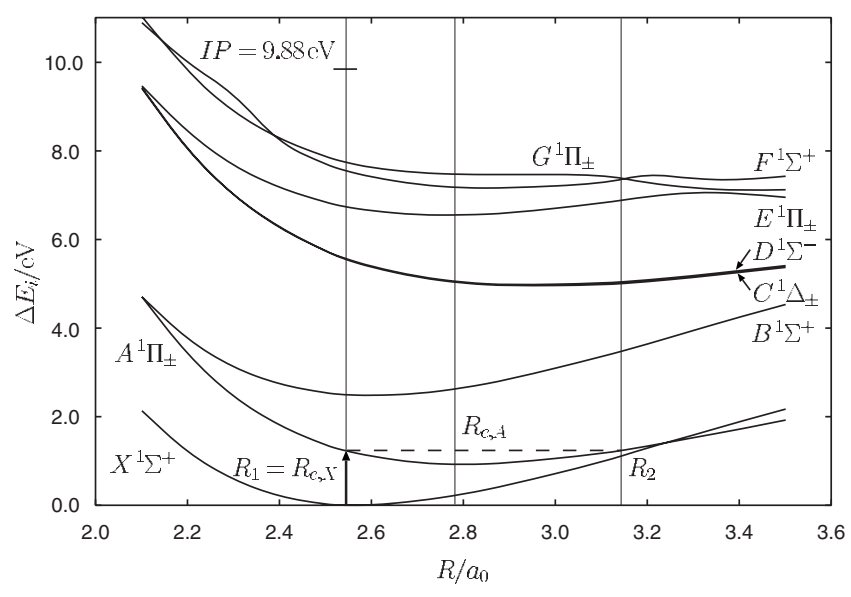

FIG. 2. Potential energy curves $\Delta E_{i}(R)=E_{i}(R)-E_{0}\left(R_{e, X}\right)$ for the ground $(i=0)$ and lowest excited $(i=1,2,3, \ldots)$ singlet states of the $\mathrm{BeO}$ molecule for bond lengths between $R=2.1 a_{0}$ and $3.5 a_{0}$. The ionization potential of the ground state $(\mathrm{IP}=9.88 \mathrm{eV})$ is marked. The arrow indicates the dipoleallowed electronic transition from the ground $X^{1} \Sigma^{+}$state into the excited $A^{1} \Pi_{+}$or $A{ }^{1} \Pi_{-}$states (corresponding to excitation by means of a right or left circularly polarized ultrashort laser pulse, respectively), see also Fig. 1. The horizontal dashed line indicates the classically allowed vibrational range in the excited $A{ }^{1} \Pi_{ \pm}$state between the classical turning points of $R_{1}=R_{e, X}=2.54 a_{0}$ and $R_{2}=3.14 a_{0}$. The equilibrium bond length of the excited state $A{ }^{1} \Pi_{ \pm}$is $R_{e, A}=2.78 a_{0}$.

$\left(1 \pi_{\overline{+}}\right) \rightarrow(5 \sigma)$ or $\left(1 \pi_{\mp}\right) \rightarrow(6 \sigma)$ but rather $(5 \sigma) \rightarrow\left(2 \pi_{ \pm}\right)$. These considerations lead us to the choice of the $\mathrm{BeO}$ molecule, which satisfies the conditions (i)-(iv) and is expected to carry strong electric ring currents and induced magnetic fields in the first excited state $A{ }^{1} \Pi_{ \pm}$.

Figure 2 shows the potential energy curves $\Delta E_{i}(R)$ $=E_{i}(R)-E_{0}\left(R_{e, X}\right)$ of the electronic ground $(i=0)$ and excited $(i=1,2,3, \ldots)$ singlet states $\left|\Psi_{i}\right\rangle$ of the BeO molecule, as calculated by means of the $\operatorname{CASSCF}(6,12) / \mathrm{CASPT} 2$ method. ${ }^{45,48-52}$ We included six electrons and 12 orbitals in the active space and used an atomic natural orbital relativistic core correlated (ANO-RCC) $5 s 4 p 2 d 1 f$ contracted basis set for both the Be- and the $\mathrm{O}$-atom, including scalar relativistic effects by means of the Douglas-Kroll transformation. ${ }^{53}$ The standard CASPT2 zeroth-order Hamiltonian was employed, ${ }^{45}$ supplemented with an imaginary level shift parameter of $0.1 E_{h}$ to prevent intruder state problems. ${ }^{54}$ Our quantum chemical calculations used the MOLCAS 6.0 program package. $^{55,56}$ Table I presents the excitation energies $\Delta E_{i}$ $=\Delta E_{i}\left(R_{e, X}\right)=E_{i}\left(R_{e, X}\right)-E_{0}\left(R_{e, X}\right)$ and the absolute values of all nonzero dipole transition matrix elements $\left|\mathbf{M}_{i j}\right|=\left|\mathbf{M}_{i j}\left(R_{e, X}\right)\right|$ $=\left|\left\langle\Psi_{i}\left(R_{e, X}\right)|\mathbf{M}| \Psi_{j}\left(R_{e, X}\right)\right\rangle\right|$ with zero Z-component of $\mathbf{M}_{i j}$. Our results were computed at the CASPT2 equilibrium bond length $R_{e, X}=2.54 a_{0}$ of the electronic ground state $X^{1} \Sigma^{+}$. This equilibrium bond length compares well with the experimental $\left(2.52 a_{0}\right),{ }^{57} \operatorname{CCSD}(\mathrm{T})\left(2.51 a_{0}\right),{ }^{41}$ and MRD-CI $\left(2.53 a_{0}\right)^{43}$ equilibrium bond lengths. The corresponding CASPT2 rotational constant at $R=R_{e, X}$ is $B_{e, X}=1.619 \mathrm{hc} \mathrm{cm} \mathrm{cm}^{-1}$, compared to the experimental value of $1.651 \mathrm{hc} \mathrm{cm}^{-1} .57$

For electric fields with nonzero $x$ - and/or $y$-components, e.g., for right circularly polarized laser pulses that propagate along the $Z$-axis of the molecule (assumed oriented), the selection rules for dipole transitions imply that all transitions that do not satisfy $\Delta M_{L}= \pm 1$ vanish, i.e., the transitions $\Sigma^{ \pm} \leftrightarrow \Pi_{ \pm}, \Pi_{+} \leftrightarrow \Delta_{+}, \Pi_{-} \leftrightarrow \Delta_{-}$, etc., are allowed, whereas other transitions, e.g., $\Sigma^{ \pm} \leftrightarrow \Sigma^{ \pm}, \quad \Pi_{ \pm} \leftrightarrow \Pi_{ \pm}, \quad \Delta_{ \pm} \leftrightarrow \Delta_{ \pm}$, $\Sigma^{ \pm} \leftrightarrow \Delta_{ \pm}, \Pi_{+} \leftrightarrow \Delta_{-}, \Pi_{-} \leftrightarrow \Delta_{+}$, etc., are forbidden.

Table I lists also the dominant electronic configurations of the electronic states of $\mathrm{BeO}$ at $R=R_{e, X}$ with the corresponding CASSCF weights. For example, the first excited degenerate state $A^{1} \Pi_{+}$is dominated by the electronic configuration ...( $\left(1 \pi_{+}\right)^{2}\left(1 \pi_{-}\right)(5 \sigma)$ with a weight of $96.1 \%$ and can be excited from the ground state $X^{1} \Sigma^{+}$with corresponding dominant electronic configuration $\cdots(1 \pi)^{4}=\ldots\left(1 \pi_{+}\right)^{2}\left(1 \pi_{-}\right)^{2}$ with weight of $67.4 \%$. The low-lying singlet excited state is therefore well characterized as corresponding to the highest occupied molecular orbital (HOMO)-LUMO transition $\left(1 \pi_{-}\right) \rightarrow(5 \sigma)$. Furthermore, the HOMO and LUMO have the LCAO-MO expansions $1 \pi_{ \pm}=c_{2 p_{ \pm}(\mathrm{O})} 2 p_{ \pm}(\mathrm{O})+c_{2 p_{+}(\mathrm{Be})} 2 p_{ \pm}(\mathrm{Be})+\cdots$ and $5 \sigma=c_{2 s(\mathrm{Be})} 2 s(\mathrm{Be})+c_{2 p_{0}(\mathrm{Be})} 2 p_{0}(\mathrm{Be})+\cdots \quad$ with weights $\left|c_{2 p_{+}(\mathrm{O})}\right|^{2}=0.96, \quad\left|c_{2 p_{ \pm}(\mathrm{Be})}\right|^{2}=0.03 \quad$ and $\quad\left|c_{2 s(\mathrm{Be})}\right|^{2}=0.67$, $\left|c_{2 p_{0}(\mathrm{Be})}\right|^{2}=0.31$, respectively. Thus, the HOMO $1 \pi_{ \pm}$is strongly localized on the $\mathrm{O}$ atom with electric ring current about the $\mathrm{O}$ nucleus, whereas the LUMO $5 \sigma$ is strongly localized on the Be atom, carrying no ring current. During the electronic excitation from the ground $X^{1} \Sigma^{+}$state to the target $A{ }^{1} \Pi_{+}$state, there is an electron transfer from the $\mathrm{O}$ - to the Be-atom, corresponding to the HOMO-LUMO transition $\left(1 \pi_{-}\right) \rightarrow(5 \sigma)$. Due to the dominant electronic configuration

TABLE I. Quantum chemistry CASSCF/CASPT2 results for $\mathrm{BeO}$ at the equilibrium bond length $R_{e, X}=2.54 a_{0}$.

\begin{tabular}{lccccccc}
\hline \hline State & Dominant configuration & $\Delta E_{i}(\mathrm{eV})$ & & & $\left|\mathbf{M}_{i j}\right| / e a_{0}{ }^{\mathrm{a}}$ & \\
\hline$X^{1} \Sigma^{+}$ & $\cdots(4 \sigma)^{2}(1 \pi)^{4}(67.4 \%)$ & 0.0000 & $A: 0.686$ & $E: 0.851$ & $G: 0.717$ & & \\
$A^{1} \Pi_{ \pm}$ & $\left(1 \pi_{+}\right) \rightarrow(5 \sigma)(96.1 \%)$ & 1.2367 & $X: 0.686$ & $B: 0.371$ & $C: 1.349$ & $D: 0.948$ & $F: 0.693$ \\
$B^{1} \Sigma^{+}$ & $(4 \sigma) \rightarrow(5 \sigma)(80.2 \%)$ & 2.4852 & $A: 0.371$ & $E: 1.154$ & $G: 0.246$ & & \\
$C^{1} \Delta_{ \pm}$ & $\left(1 \pi_{+}\right) \rightarrow\left(2 \pi_{ \pm}\right)(95.7 \%)$ & 5.5636 & $A: 1.349$ & $E: 0.074$ & $G: 0.518$ & & \\
$D^{1} \Sigma^{-}$ & $\left(1 \pi_{-}\right) \rightarrow\left(2 \pi_{+}\right)(48.4 \%)$ & 5.5773 & $A: 0.948$ & $E: 0.004$ & $G: 0.400$ & & \\
& $\left(1 \pi_{+}\right) \rightarrow\left(2 \pi_{-}\right)(48.4 \%)$ & & & & & & \\
$E^{1} \Pi_{ \pm}$ & $(4 \sigma) \rightarrow\left(2 \pi_{ \pm}\right)(95.5 \%)$ & 6.7417 & $X: 0.851$ & $B: 1.154$ & $C: 0.074$ & $D: 0.004$ & $F: 0.275$ \\
$F^{1} \Sigma^{+}$ & $\left(1 \pi_{+}\right) \rightarrow\left(2 \pi_{+}\right)(31.0 \%)$ & 7.5513 & $A: 0.693$ & $E: 0.275$ & $G: 0.145$ & & \\
& $\left(1 \pi_{-}\right) \rightarrow\left(2 \pi_{-}\right)(31.0 \%)$ & & & & & & \\
$G^{1} \Pi_{ \pm}$ & $\left(1 \pi_{+}\right) \rightarrow(6 \sigma)(94.7 \%)$ & 7.7462 & $X: 0.717$ & $B: 0.246$ & $C: 0.518$ & $D: 0.400$ & $F: 0.145$ \\
\hline \hline
\end{tabular}


$\ldots\left(1 \pi_{+}\right)^{2}\left(1 \pi_{-}\right)(5 \sigma)$ of the excited state, the net electric ring current is dominated by that of the $1 \pi_{+}$orbital with weight of at least $96.1 \%$.

Considering next the properties of the ground electronic, $X^{1} \Sigma^{+}$, state, we find that the CASPT2 permanent electric dipole moment at $R=R_{e, X}$ is $\mu\left(R_{e, X}\right)=\left|\mathbf{M}_{00}\left(R_{e, X}\right)\right|$ $=\left|\left\langle\Psi_{0}\left(R_{e, X}\right)|\mathbf{M}| \Psi_{0}\left(R_{e, X}\right)\right\rangle\right|=6.50 \mathrm{D}$, somewhat higher than the early experimental estimation of 5.766 D. ${ }^{57}$ Other reported values, including the $\operatorname{CCSD}(\mathrm{T})$ result $(6.25 \mathrm{D})$ (Ref. $41)$ and the MRD-CI result $(6.14 \mathrm{D}),^{43}$ are in close agreement with our CASPT2 result. Note that the direction of the dipole vector is from the $\mathrm{O}$ - to the $\mathrm{Be}$-atom because the partial charges of $\mathrm{Be}$ and $\mathrm{O}$ are positive and negative, respectively. The first ionization potential of $\mathrm{BeO}$ at $R=R_{e, X}$ is $\mathrm{IP}=9.88 \mathrm{eV}$ at the CASPT2 level, which compares with the experimental value of $10.1 \pm 0.4 \mathrm{eV}$ (Ref. 58) better than the MRD-CI value, $10.64 \mathrm{eV}^{43}$

The CASPT2 equilibrium bond length of the excited state $A{ }^{1} \Pi_{ \pm}$is $R_{e, A}=2.78 a_{0}$ in agreement with the experimental $\left(2.76 a_{0}\right)$ (Ref. 57) and MRD-CI (2.79a $a_{0}$ (Ref. 43) values. The corresponding CASPT2 rotational constant at $R=R_{e, A}$ is $B_{e, A}=1.351 h c \mathrm{~cm}^{-1}$. The equilibrium displacement of the potential curves of the ground $X^{1} \Sigma^{+}$and excited $A^{1} \Pi_{ \pm}$states is thus $\Delta R_{e}=R_{e, A}-R_{e, X}=0.26 a_{0}$. Due to this displacement, there are dominant Franck-Condon (FC)-type electronic excitations from the vibrational ground state $\nu=0$ in the electronic $X^{1} \Sigma^{+}$state to the vibrational excited states $v^{\prime}=0,1,2,3,4$, and 5 in the electronic $A^{1} \Pi_{ \pm}$state with weights of $0.12,0.23,0.25,0.18,0.12$, and 0.06 , respectively. These weights are estimated using experimental spectroscopic constants of the potential curves $X^{1} \Sigma^{+}$and
$A^{1} \Pi_{ \pm} \cdot{ }^{57}$ The classical turning points due to the vibration of the $\mathrm{BeO}$ molecule in the excited state $A^{1} \Pi_{ \pm}$after the electronic FC excitation $X^{1} \Sigma^{+} \rightarrow A^{1} \Pi_{ \pm}$are $\bar{R}_{1}=R_{e, X}=2.54 a_{0}$ and $R_{2}=3.14 a_{0}$.

\section{ORIENTATION OF THE BeO MOLECULE}

We consider ground state $\mathrm{BeO}$ molecules at a rotational temperature $T$, hence the initial state is the $X^{1} \Sigma^{+}(\nu=0)$ vibronic state with a Boltzmann distribution of the rotational levels $|J M\rangle \quad\left(J=0,1,2, \ldots, J_{\max }\right.$ and $M=-J,-J+1, \ldots$, $J-1, J)$. Rotation-vibration coupling is neglected, as we consider time scales much shorter than the time scales at which the effects of such interactions are observable. ${ }^{59}$ In the rigidrotor limit, the ground state rotational energies are $E_{X}^{J}=B_{e, X} J(J+1)$ and the Boltzmann weights are calculated as

$$
P(J)=\frac{e^{-B_{e, X} J(J+1) / k_{B} T}}{\sum_{J=0}^{J_{\max }}(2 J+1) e^{-B_{e, X} J(J+1) / k_{B} T}} .
$$

For the $\mathrm{BeO}$ molecule at $T=1 \mathrm{~K}$, the first three Boltzmann weights are $P(0)=0.972, P(1)=0.009$, and $P(2)=10^{-6}$.

Linearly polarized half cycle pulses (HCPs) have been previously shown to give rise to molecular orientation ${ }^{60-62}$ (see also the introduction of Ref. 63), but do not satisfy the condition of the far-field approximation of Maxwell's equations, ${ }^{64,65}$ i.e.,

$$
\int_{-\infty}^{\infty} \mathbf{E}(t) d t=0
$$

Here, we construct a linearly polarized electric field of the form

$$
\mathbf{E}_{l}(t)= \begin{cases}0 & (t \leq 0) \\ -\mathcal{E}_{1, l} \cos ^{2}\left(\frac{\omega_{l}\left(t-t_{p, l}\right)}{2}\right) \sin \left(\omega_{l}\left(t-t_{p, l}\right)\right) \mathbf{e}_{z} & \left(0 \leq t \leq t_{p, l}\right) \\ -\mathcal{E}_{2, l}\left(1-e^{\left.-\left(t-t_{p, l}\right) / \tau_{1, l}\right)} e^{-\left(t-t_{p, l}\right) / \tau_{2, l}} \mathbf{e}_{z}\right. & \left(t \geq t_{p, l}\right),\end{cases}
$$

where $\mathcal{E}_{1, l}, \mathcal{E}_{2, l}$ are electric field amplitudes, $\omega_{l}$ is the carrier frequency, $t_{p, l}=\pi / \omega_{l}$ is the pulse duration of the first part of the laser pulse, $\tau_{2, l}, \tau_{2, l}$ are the switch-on and switch-off times of the second part of the laser pulse, respectively, and the index $l$ denotes linear polarization. The condition that the electric field (3) is smooth at $t=t_{p, l}$ leads to

$$
\tau_{1, l}=\frac{\mathcal{E}_{2, l}}{\omega_{l} \mathcal{E}_{1, l}},
$$

and the condition (2) yields

$$
\tau_{2, l}=\frac{1}{2 \omega_{l}^{2} \tau_{1, l}}+\sqrt{\frac{1}{4 \omega_{l}^{4} \tau_{1, l}^{2}}+\frac{1}{\omega_{l}^{2}}} .
$$

The first part of the laser pulse $\left(0 \leq t \leq t_{p, l}\right)$ is similar to the $\mathrm{HCP}$, and its magnitude exceeds that of the second part of the laser pulse $\left(t \geq t_{p, l}\right),\left|\mathcal{E}_{1, l}\right|>\left|\mathcal{E}_{2, l}\right|$. The latter half has a long tail that ensures that the condition (2) would be satisfied. To provide a useful compromise between a large amplitude ratio and an acceptable switch-off time, we use in the present work $\mathcal{E}_{1, l} / \mathcal{E}_{2, l}=10$. Since the dipole vector points from the $\mathrm{O}$ to the $\mathrm{Be}$-atom, we choose the laser parameters so as to optimize the orientation of the $\mathrm{BeO}$ molecule along the negative space-fixed $z$-axis (see also Fig. 1), hence the amplitudes $\mathcal{E}_{1, l}$ and $\mathcal{E}_{2, l}$ are taken to be negative. With the amplitude $\mathcal{E}_{1, l}$ and carrier frequency $\omega_{l}$ optimized to yield tight (inverted) orientation of the $\mathrm{BeO}$ molecule, we find the values $\mathcal{E}_{1, l}=-100.0 \mathrm{MV} \mathrm{m}^{-1}$ and $\hbar \omega_{l}=12.0 h c \mathrm{~cm}^{-1}$. Thus $\mathcal{E}_{2, l}=-10.0 \mathrm{MV} \mathrm{m}^{-1}$, the pulse duration is $t_{p, l}=\pi / \omega_{l}$ $=1.39 \mathrm{ps}$, and the switch-on and switch-off times [Eqs. (4) and (5)] are estimated as $\tau_{1, l}=44.2$ fs and $\tau_{2, l}=4.47 \mathrm{ps}$. The maximum intensity of the laser pulse is 


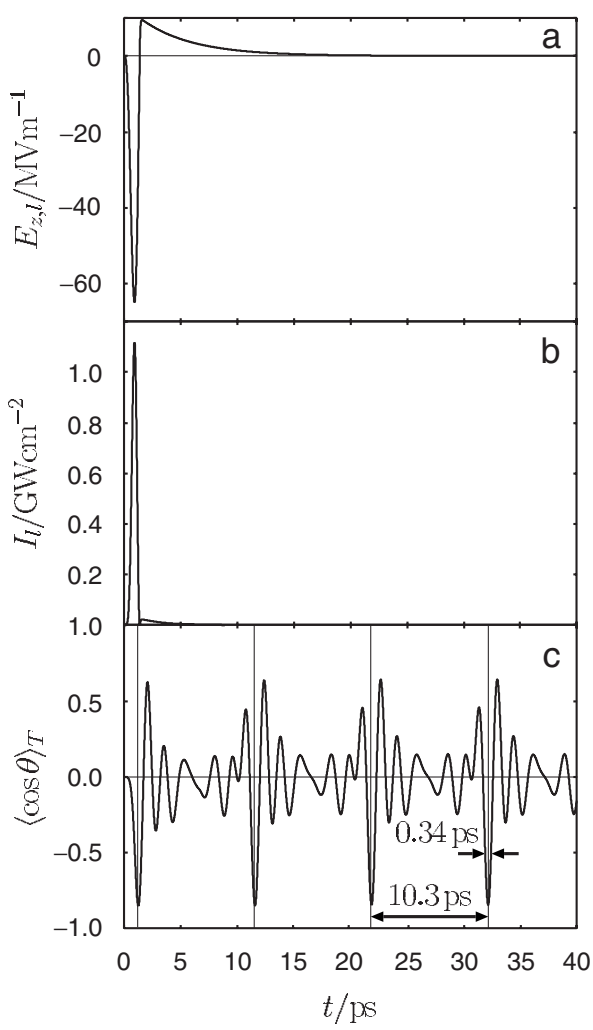

FIG. 3. $z$-component of the time-dependent electric field $E_{z, l}(t)$ (panel a) and its intensity $I_{l}(t)=c \varepsilon_{0}\left|\mathbf{E}_{l}(t)\right|^{2}$ (panel b). The pulse parameters in Eq. (3) are $\mathcal{E}_{1, l}=-100 \mathrm{MV} \mathrm{m}^{-1}, \quad \mathcal{E}_{2, l}=-10 \mathrm{MV} \mathrm{m}^{-1}, \hbar \omega_{l}=12.0 h c \mathrm{~cm}^{-1}, t_{p}=1.39 \mathrm{ps}$, $\tau_{1, l}=44.2 \mathrm{fs}$, and $\tau_{2, l}=4.47 \mathrm{ps}$. Panel c shows the thermally averaged timedependent expectation value $\langle\cos \theta\rangle_{T}(t)$ at $T=1 \mathrm{~K}$. The orientation revivals (marked by vertical lines) are spaced by the rotational revival time $\tau_{\mathrm{rev}, X}$ $=10.30 \mathrm{ps}$ of the electronic ground state $X^{1} \Sigma^{+}$. The orientation duration [taken to be the time at which $\left|\langle\cos \theta\rangle_{T}(t)\right|>0.5$ ] is 0.34 ps.

$I_{\text {max }, l}=c \varepsilon_{0} \max \left|\mathbf{E}_{l}(t)\right|^{2}=1.12 \mathrm{GV} \mathrm{cm}^{-2}$ since $\max \left|\mathbf{E}_{l}(t)\right|$ $=65.0 \mathrm{MV} \mathrm{m}^{-1}<\left|\mathcal{E}_{1, l}\right|$. Figures 3(a) and 3(b) present the $z$-component of the time-dependent electric field $\mathbf{E}_{l}(t)=E_{z, l}(t) \mathbf{e}_{z} \quad[$ Eq. (3) $]$ and the corresponding timedependent intensity $I_{l}(t)=c \varepsilon_{0}\left|\mathbf{E}_{l}(t)\right|^{2}$, respectively. This laser pulse is very similar to the experimental laser pulse shown in Fig. 2 of Ref. 66, i.e., with almost the same pulse shape and duration, but with lower intensity.

The carrier frequency $\hbar \omega_{l}=12.0 h c \mathrm{~cm}^{-1}$ used here is small with respect to the experimental vibration frequency of the electronic ground state $\hbar \omega_{e, X}=1487.3 h c \mathrm{~cm}^{-1}$ (Ref. 57) and with respect to the excitation frequency of the first excited electronic state $A{ }^{1} \Pi_{ \pm}$at the equilibrium bond length, $\Delta E_{1}\left(R_{e, X}\right)=1.2367 \mathrm{eV}=9974.7 h \mathrm{~cm}^{-1}$. Thus, the laser frequency $\omega_{l}$ is far off resonant with all vibrational and electronic excitations and hence the molecule remains in the electronic and vibrational ground states, $X^{1} \Sigma^{+}(\nu=0)$, while the moderately intense field populates a broad rotational wavepacket. The time-dependent Schrödinger equation is written within the electric dipole approximation as

$$
i \hbar \frac{\partial}{\partial t}\left|\Psi_{\text {rot }}(t)\right\rangle=\left(H_{\text {rot }}-\mu\left(R_{e, X}\right) \cos \theta E_{z, l}(t)\right)\left|\Psi_{\text {rot }}(t)\right\rangle,
$$

where $H_{\text {rot }}$ is the rigid-rotor rotational Hamiltonian,

$$
H_{\mathrm{rot}}=B_{e, X} \hat{J}^{2}
$$

and $\hat{J}$ is the total angular momentum operator. The timedependent rotational wavepacket $\left|\Psi_{\text {rot }}(t)\right\rangle$ is expanded in terms of the rotational eigenstates $|J M\rangle$ of $H_{\text {rot }}$ as

$$
\left|\Psi_{\mathrm{rot}}^{J_{i} M_{i}}(t)\right\rangle=\sum_{J=0}^{J_{\max }} \sum_{M=-J}^{J} C_{J M}^{J_{j} M_{i}}(t)|J M\rangle e^{-i E_{X}^{J}{ }^{t / \hbar}}
$$

with the initial condition

$$
\left|\Psi_{\text {rot }}^{J_{i} M_{i}}(t=0)\right\rangle=\left|J_{i} M_{i}\right\rangle \text {. }
$$

Inserting the ansatz (8) and (9) into Eq. (6), one obtains a set of coupled equations for the time-dependent expansion coefficients,

$$
\begin{aligned}
i \hbar \frac{d}{d t} C_{J M}^{J_{i} M_{i}}(t)= & -\mu\left(R_{e, X}\right) E_{z, l}(t) \sum_{J^{\prime}=0}^{J_{\max }} \sum_{M^{\prime}=-J^{\prime}}^{J^{\prime}}\left\langle J M|\cos \theta| J^{\prime} M^{\prime}\right\rangle \\
& \times e^{-i\left(E_{X}^{J^{\prime}}-E_{X}^{J}\right) t / \hbar} C_{J^{\prime} M^{\prime}}^{J_{i} M_{i}}(t),
\end{aligned}
$$

with initial condition

$$
C_{J M}^{J_{i} M_{i}}(t=0)=\delta_{J J_{i}} \delta_{M M_{i}} .
$$

The selection rules $J^{\prime}=J \pm 1$ and $M^{\prime}=M$ simplify the differential equations (10) as

$$
\begin{aligned}
i \hbar \frac{d}{d t} C_{J M_{i}}^{J_{i} M_{i}}(t)= & -\mu\left(R_{e, X}\right) E_{z, l}(t) \sum_{J^{\prime}=0}^{J_{\max }}\left\langle J M_{i}|\cos \theta| J^{\prime} M_{i}\right\rangle \\
& \times e^{-i\left(E_{X}^{J^{\prime}}-E_{X}^{J}\right) t / \hbar} C_{J^{\prime} M_{i}}^{J_{i} M_{i}}(t)
\end{aligned}
$$

and

$$
C_{J M}^{J_{i} M_{i}}(t)=0
$$

for $M \neq M_{i}$. The time-dependent rotational wavepacket (8) is thus

$$
\left|\Psi_{\text {rot }}^{J_{i} M_{i}}(t)\right\rangle=\sum_{J=0}^{J_{\max }} C_{J M_{i}}^{J_{i} M_{i}}(t)\left|J M_{i}\right\rangle e^{-i E_{X}^{J} t / \hbar} .
$$

The matrix elements $\left\langle J M_{i}|\cos \theta| J^{\prime} M_{i}\right\rangle$ for $J^{\prime}=J \pm 1$ are given explicitly as ${ }^{67,68}$

$$
\begin{aligned}
\left\langle J M_{i}|\cos \theta| J^{\prime} M_{i}\right\rangle= & (-1)^{M_{i}} \sqrt{(2 J+1)\left(2 J^{\prime}+1\right)} \\
& \times\left(\begin{array}{ccc}
J & 1 & J^{\prime} \\
M_{i} & 0 & -M_{i}
\end{array}\right)\left(\begin{array}{ccc}
J & 1 & J^{\prime} \\
0 & 0 & 0
\end{array}\right) .
\end{aligned}
$$

Equations (12) and (11) are solved by means of the Runge-Kutta method of fourth order (Simpson rule $)^{69}$ with a time step size of $\Delta t=5$ fs, with which the norm of the timedependent coefficients is converged to within $10^{-6}$.

To quantify the degree of molecular orientation, we calculate the time-dependent expectation value of $\cos \theta$ as 


$$
\begin{aligned}
\langle\cos \theta\rangle_{J_{i} M_{i}}(t)= & \left\langle\Psi_{\text {rot }}^{J_{i} M_{i}}(t)|\cos \theta| \Psi_{\text {rot }}^{J_{i} M_{i}}(t)\right\rangle \\
= & \sum_{J, J^{\prime}=0}^{J_{\max }}\left(C_{J M_{i}}^{J_{i} M_{i}}(t)\right)^{*} C_{J^{\prime} M_{i}}^{J_{j} M_{i}}(t) \\
& \times\left\langle J M_{i}|\cos \theta| J^{\prime} M_{i}\right\rangle e^{-i\left(E_{X}^{J^{\prime}}-E_{X}^{J}\right) t / \hbar} .
\end{aligned}
$$

The corresponding thermally averaged time-dependent expectation value is given as a Boltzmann average over the initial state-selected values of Eq. (16),

$$
\langle\cos \theta\rangle_{T}(t)=\sum_{J_{i}=J_{i, \min }}^{J_{i, \max }} P\left(J_{i}\right) \sum_{M_{i}=-J_{i}}^{J_{i}}\langle\cos \theta\rangle_{J_{i} M_{i}}(t),
$$

where, at $T=1 \mathrm{~K}, J_{i, \min }=0$ and $J_{i, \max }=2$. Positive and negative signs of $\langle\cos \theta\rangle_{T}(t)$ correspond to orientation in the positive and negative $z$-directions, respectively, and $\left|\langle\cos \theta\rangle_{T}(t)\right|$ $=1$ corresponds to the idealized limit of perfect orientation. Figure 3(c) shows the thermally averaged expectation value $\langle\cos \theta\rangle_{T}(t)$ at $T=1 \mathrm{~K}$ for the optimized laser pulse. The orientation takes a minimum value of -0.850 shortly after the turn off, implying strong orientation of the molecule in the negative $z$-direction. As expected, an early dephasing is followed at long times by a series of revivals at which the orientation is periodically reconstructed at intervals of the rotational revival time. ${ }^{70}$ For the electronic ground state of the $\mathrm{BeO}, \tau_{\mathrm{rev}, X}=\pi \hbar / B_{e, X}=10.30$ ps. The orientation durations are 0.34 and $0.17 \mathrm{ps}$ for absolute values of $\langle\cos \theta\rangle_{T}(t)$ larger than 0.5 and 0.8 , respectively.

The time- and angle-resolved distribution of the rotational wavepacket for a rotationally selected parent state $\left|J_{i} M_{i}\right\rangle$ is obtained as an integral over the (isotropic) $\varphi$ distribution,

$$
\left|\Psi_{\text {rot }}^{J_{i} M_{i}}(\theta, t)\right|^{2}=\int_{0}^{2 \pi}\left|\Psi_{\text {rot }}^{J_{i} M_{i}}(\theta, \varphi, t)\right|^{2} d \varphi
$$

and the corresponding thermally averaged time-dependent angular distribution is

$$
\left|\Psi_{\mathrm{rot}}(\theta, t)\right|_{T}^{2}=\sum_{J_{i}=J_{i, \text { min }}}^{J_{i, \text { max }}} P\left(J_{i}\right) \sum_{M_{i}=-J_{i}}^{J_{i}}\left|\Psi_{\mathrm{rot}}^{J_{i} M_{i}}(\theta, t)\right|^{2} .
$$

Figure 4 shows the normalized angular distribution $\left|\Psi_{\text {rot }}(\theta, t)\right|_{T}^{2} \sin \theta(T=1 \mathrm{~K})$ at the instance of almost perfect negative orientation of the $\mathrm{BeO}$ molecule $t=t^{*}=21.88 \mathrm{ps}$ and the instances at which the peak orientation revives $t=t^{*}+n \tau_{\mathrm{rev}, X}(n=0,1,2, \ldots)$, where the angular distribution is focused in the $160^{\circ}-180^{\circ}$ range. Also shown in Fig. 4 is the initial angular distribution at $t=0$.

\section{ELECTRIC RING CURRENT AND INDUCED MAGNETIC FIELD IN ORIENTED BeO MOLECULES}

In this section we assume that the $\mathrm{BeO}$ molecule in the electronic ground state $X^{1} \Sigma^{+}$is perfectly oriented along the $z$-axis, a condition that can be approximately achieved using the method described in Sec. III, where the almost perfect orientation attained at the revival times is shown to persist for about 170 fs through which $\left|\langle\cos \theta\rangle_{T}\right| \geq 0.8$. This time is much larger than the pulse duration $\left(t_{p, c}=21.2 \mathrm{fs}\right)$ of the

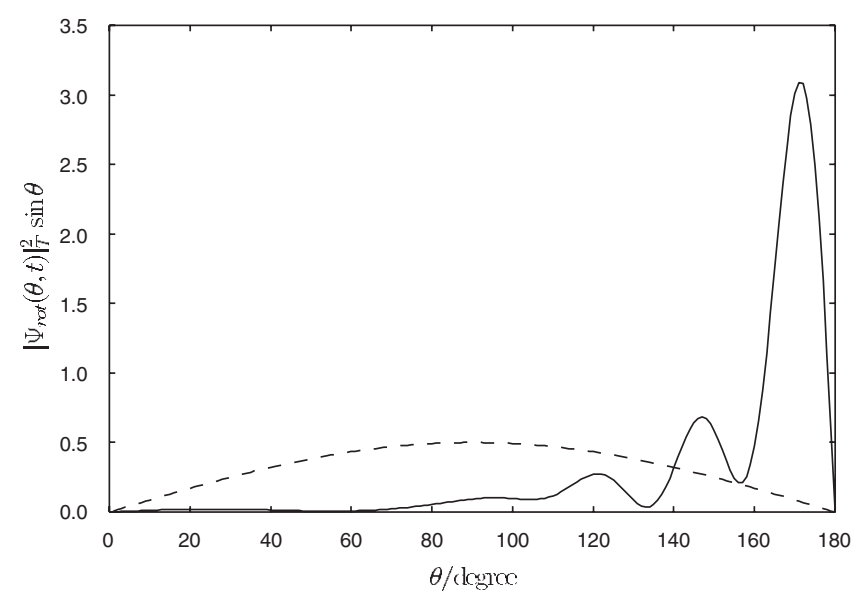

FIG. 4. Thermally averaged normalized angular distribution of the rotational wavepacket $\quad\left|\Psi_{\text {rot }}(\theta, t)\right|_{T}^{2} \sin \theta \quad(T=1 \mathrm{~K}) \quad$ at times $t=t^{*}+n \tau_{\text {rev }, X}$ $(n=0,1,2, \ldots)$ (bold curve) compared with the initial angular distribution (dashed curve).

subsequent circularly polarized laser pulse that triggers electric ring currents. The effects of deviations from perfect orientation are discussed at the end of this section. The circularly polarized field is thus centered at a selected revival of the rotational wavepacket, with its propagation direction along the alignment field polarization direction, see Fig. 1. The excitation electric field is defined through the vector potential, ${ }^{19,24}$

$$
\mathbf{A}_{c \pm}(t)=-\frac{\mathcal{E}_{c}}{\omega_{c}} s\left(t-t^{*}\right)\left(\begin{array}{c}
\sin \left(\omega_{c}\left(t-t^{*}\right)\right) \\
\mp \cos \left(\omega_{c}\left(t-t^{*}\right)\right) \\
0
\end{array}\right),
$$

with which the time-dependent right $(+)$ or left $(-)$ circularly polarized electric field is written,

$$
\begin{aligned}
\mathbf{E}_{c \pm}(t)=-\frac{d}{d t} \mathbf{A}_{c \pm}(t)= & \mathcal{E}_{c} s\left(t-t^{*}\right)\left(\begin{array}{c}
\cos \left(\omega_{c}\left(t-t^{*}\right)\right) \\
\pm \sin \left(\omega_{c}\left(t-t^{*}\right)\right) \\
0
\end{array}\right) \\
& +\frac{\mathcal{E}_{c}}{\omega_{c}} \dot{s}\left(t-t^{*}\right)\left(\begin{array}{c}
\sin \left(\omega_{c}\left(t-t^{*}\right)\right) \\
\mp \cos \left(\omega_{c}\left(t-t^{*}\right)\right) \\
0
\end{array}\right),
\end{aligned}
$$

with amplitude $\mathcal{E}_{c}$, carrier frequency $\omega_{c}$, and Gaussian-type envelope, ${ }^{24}$

$$
s\left(t-t^{*}\right)= \begin{cases}\cos ^{20}\left(\frac{\pi\left(t-t^{*}\right)}{t_{p, c}}\right) & \text { for }\left|t-t^{*}\right| \leq \frac{t_{p, c}}{2} \\ 0 & \text { for }\left|t-t^{*}\right|>\frac{t_{p, c}}{2},\end{cases}
$$

where $t_{p, c}$ is the total pulse duration. For the time propagation of the electronic states the initial and final times are set to $t_{0}=t^{*}-t_{p, c} / 2$ and $t_{f}=t^{*}+t_{p, c} / 2$, respectively, where $t^{*}$ is the instant of best orientation of the molecule in the negative $z$-direction (or any of the subsequent times at which the peak orientation is reconstructed). Note that the circularly polarized electric field (21) satisfies the condition (2) automatically, and that for few-cycle laser pulses the second term of 
Eq. (21) cannot be neglected. The time-dependent intensity is calculated as $I_{c}(t)=c \varepsilon_{0}\left|\mathbf{E}_{c \pm}(t)\right|^{2}$, which is independent of the sense of the polarization. The maximum of the intensity and the full width at half maximum are $I_{\max , c}=\max I_{c}(t)$ and $\tau_{c}$, respectively. Note that for few-cycle laser pulses the corresponding simple relations for manycycle laser pulses given in Ref. 19, i.e., $I_{\max , c}=c \varepsilon_{0} \mathcal{E}_{c}^{2}$ and $\tau_{c}=0.118 t_{p, c}$, do not apply.

The laser parameters of the right $(+)$ or left $(-)$ circularly polarized laser pulse (21) are determined in order to achieve an almost perfect population transfer from the electronic ground $X^{1} \Sigma^{+}$to excited $A^{1} \Pi_{+}$or $A^{1} \Pi_{-}$states of the $\mathrm{BeO}$ molecule, respectively. ${ }^{19}$ The pulse duration $\tau_{c}=2.74 \mathrm{fs}$ is taken to be much shorter than the vibrational period in the excited state $A{ }^{1} \Pi_{ \pm}\left[\tau_{\mathrm{vib}, A}=29.2\right.$ fs (Ref. 57)], and therefore we can assume that the nuclei are frozen during the FC electronic excitation. The optimized laser parameters of the circularly polarized laser pulse are $\mathcal{E}_{c}=10.05 \mathrm{GV} \mathrm{m}^{-1}$ and $\hbar \omega_{c}=1.04 \mathrm{eV}=8388.2 h \mathrm{~cm}^{-1}$, with a corresponding intensity maximum of $I_{\max , c}=I_{c}\left(t^{*}\right)=26.8 \mathrm{TW} \mathrm{cm}{ }^{-2}$ and optical cycle duration of $\Delta t_{c}=2 \pi / \omega_{c}=3.98$ fs. Figures $5(\mathrm{a})$ and 5(b) show, respectively, the $x$ - and $y$-components of the timedependent electric field $\mathbf{E}_{c+}(t)=E_{x, c}(t) \mathbf{e}_{x}+E_{y, c}(t) \mathbf{e}_{y}$ [Eq. (21)] centered at $t^{*}$ for the right circularly polarized laser pulse, see also Fig. 1. The corresponding time-dependent intensity $I_{c}(t)$ is shown in Fig. 5(c).

The laser-driven electron dynamics of the $\mathrm{BeO}$ molecule at the equilibrium bond length $R_{e, X}=2.54 a_{0}$ is described, within electric dipole approximation, by the time-dependent electronic Schrödinger equation,

$$
i \hbar \frac{\partial}{\partial t}\left|\Psi_{\mathrm{el}}\left(R_{e, X}, t\right)\right\rangle=\left(H_{\mathrm{el}}-\mathbf{M} \cdot \mathbf{E}_{c \pm}(t)\right)\left|\Psi_{\mathrm{el}}\left(R_{e, X}, t\right)\right\rangle,
$$

where $H_{\mathrm{el}}$ and $\mathbf{M}$ are the electronic Hamiltonian and dipole operator, respectively. In an analogous way to that described in Sec. III, the time-dependent electronic state $\left|\Psi_{\mathrm{el}}\left(R_{e, X}, t\right)\right\rangle$ is expanded in terms of electronic eigenstates $\left|\Psi_{i}\left(R_{e, X}\right)\right\rangle$ of $H_{\mathrm{el}}$ with electronic quantum numbers $i=0,1,2, \ldots, i_{\max }$ and corresponding eigenenergies $E_{i}\left(R_{e, X}\right)$, thus

$$
\left|\Psi_{\mathrm{el}}\left(R_{e, X}, t\right)\right\rangle=\sum_{i=0}^{i_{\max }} C_{i}(t)\left|\Psi_{i}\left(R_{e, X}\right)\right\rangle e^{-i E_{i}\left(R_{e, X}\right)\left(t-t_{0}\right) / \hbar}
$$

with initial condition

$$
\left|\Psi_{\mathrm{el}}\left(R_{e, X}, t=t_{0}\right)\right\rangle=\left|\Psi_{0}\left(R_{e, X}\right)\right\rangle=\left|\Psi_{X} \Sigma_{\Sigma^{+}}\left(R_{e, X}\right)\right\rangle,
$$

where $i_{\max }+1=12$ is the number of electronic states of the $\mathrm{BeO}$ molecule considered, see also Table I. Since the ionization potential of the $\mathrm{BeO}$ molecule $(\mathrm{IP}=9.88 \mathrm{eV})$ is large compared to the excitation energy of the target $A{ }^{1} \Pi_{ \pm}$state $\left(\Delta E_{1}=1.24 \mathrm{eV}\right)$, and the maximum of the intensity of the laser pulse is well below the Keldish limit, ${ }^{71}$ it can be safely assumed that continuum states describing ionization need not be included in the expansion [Eq. (24)]. Inserting the ansatz (24) and (25) into Eq. (23), we obtain a set of coupled equations for the time-dependent expansion coefficients,

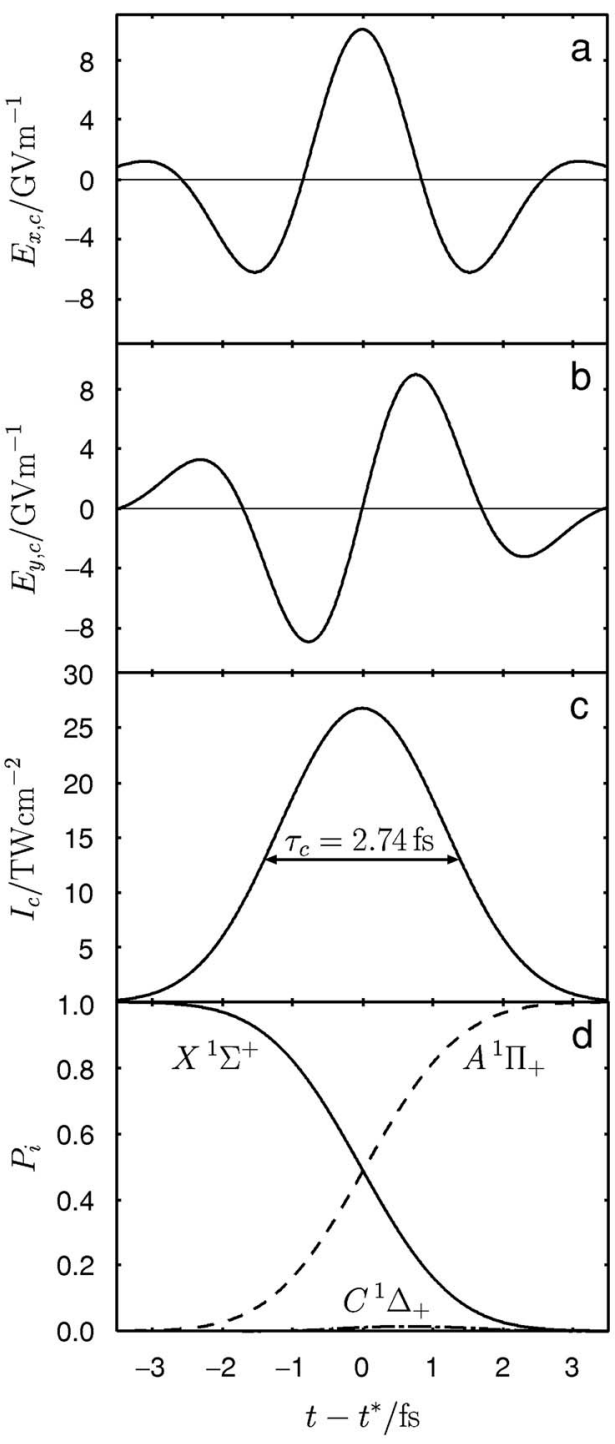

FIG. 5. $x$ - and $y$-components of the time-dependent electric field $E_{x, c}(t)$ and $E_{y, c}(t)$ (panels a and $\mathrm{b}$, respectively) and its intensity $I_{c}(t)=c \varepsilon_{0}\left|\mathbf{E}_{c+}(t)\right|^{2}$ (panel c) for the optimized right circularly polarized ultrashort few-cycle laser pulse shown in Fig. 1. Panel d shows the corresponding population dynamics in the electronic $X^{1} \Sigma^{+}, A{ }^{1} \Pi_{+}$, and $C^{1} \Delta_{+}$states at the equilibrium bond length $R=R_{e, X}=2.54 a_{0}$ of the $\mathrm{BeO}$ molecule.

$$
i \hbar \frac{d}{d t} C_{i}(t)=-\mathbf{E}_{c \pm}(t) \cdot \sum_{j=0}^{i_{\max }} \mathbf{M}_{i j} e^{-i\left(\Delta E_{j}-\Delta E_{i}\right)\left(t-t_{0}\right) / \hbar} C_{j}(t),
$$

with initial condition

$$
C_{i}\left(t=t_{0}\right)=\delta_{0 i}
$$

for $i=0,1,2, \ldots, i_{\max }$. The absolute values of the dipole matrix elements, $\left|\mathbf{M}_{i j}\right|=\left|\mathbf{M}_{i j}\left(R_{e, X}\right)\right|$, and the excitation energies, $\Delta E_{i}=\Delta E_{i}\left(R_{e, X}\right)=E_{i}\left(R_{e, X}\right)-E_{0}\left(R_{e, X}\right)$, for the $\mathrm{BeO}$ molecule are given in Table I. The selection rules for electronic excitations are described in Sec. II. Equations (26) and (27) are solved by means of the Runge-Kutta method of fourth order (Simpson rule) $^{69}$ with a time step size of $\Delta t=1$ as.

The time-dependent populations $P_{i}(t)$ of the electronic states $\left|\Psi_{i}\left(R_{e, X}\right)\right\rangle$ are calculated as 


$$
P_{i}(t)=\left|C_{i}(t)\right|^{2} \quad\left(i=0,1,2, \ldots, i_{\max }\right),
$$

and the dominant populations of the electronic states $X^{1} \Sigma^{+}$, $A^{1} \Pi_{+}$and also $C^{1} \Delta_{+}$are shown in Fig. 5(d) for the optimized right circularly polarized laser pulse illustrated in Figs. 1 and 5(a)-5(c). The near-resonance laser pulse induces almost perfect population transfer from the ground $X^{1} \Sigma^{+}$to the target $A^{1} \Pi_{+}$states with the final populations, $P_{X^{1} \Sigma^{+}}\left(t_{f}\right)$ $=5 \times 10^{-7}$ and $P_{A}{ }^{1} \Pi_{+}\left(t_{f}\right)=1-6 \times 10^{-7}$. This corresponds to the dominant HOMO-LUMO transition $\left(1 \pi_{-}\right) \rightarrow(5 \sigma)$. The few-cycle laser pulse can induce the competing nearresonant transition $X^{1} \Sigma^{+} \rightarrow A^{1} \Pi_{-}$, but the corresponding population is small due to the oscillatory nature of the nonrotating wave terms in Eq. (26). For instance, $\max P_{A^{1} \Pi}(t)=0.0077$ and $P_{A}{ }^{1} \Pi\left(t_{f}\right)=2 \times 10^{-8}$. The strongest dipole-allowed transition $A^{1} \Pi_{+} \rightarrow C^{1} \Delta_{+}$induces a small transient population in the excited $C^{1} \Delta_{+}$state, $\max P_{C{ }^{1} \Delta_{+}}(t)=0.0161$, and $P_{C{ }^{1} \Delta_{+}}\left(t_{f}\right)=8 \times 10^{-8}$. It corresponds to the dominant transition $(5 \sigma) \rightarrow\left(2 \pi_{+}\right)$, see also Table I. All other states have negligible populations.

After the laser pulse, i.e., after the $\mathrm{FC}$ electronic excitation from the ground $X^{1} \Sigma^{+}$to target $A{ }^{1} \Pi_{+}$states, the vibrational wavepacket in the excited state $A{ }^{1} \Pi_{+}$begins to oscillate between the classical turning points $R_{1}=R_{e, X}=2.54 a_{0}$ and $R_{2}=3.14 a_{0}$, see Fig. 2. The final electronic state $\left|\Psi_{A}{ }^{1} \Pi_{+}(R)\right\rangle$ is now dependent on the bond length $R$ of the $\mathrm{BeO}$ molecule and represents the $R$-dependent toroidal electric ring current of the electron about the molecular axis. We will show that the electric ring current and induced magnetic field are almost independent of the bond length $R$ between $R_{1}$ and $R_{2}$, thus we can neglect the time dependence of the electric ring current due to the vibration of the molecule in the excited state $A^{1} \Pi_{+}$. Furthermore, the excited state $A{ }^{1} \Pi_{+}$represents the dominant HOMO-LUMO transition $\left(1 \pi_{-}\right) \rightarrow(5 \sigma)$ with weight of $96.0 \% \pm 0.6 \%$ for bond lengths between $R_{1}$ and $R_{2}$. Thus, the net electric ring current of the state $A{ }^{1} \Pi_{+}$is dominated by the electric ring current of the $1 \pi_{+}$orbital with the same weight. The $R$-dependent electronic current density of the $A^{1} \Pi_{+}$state can be approximated by that of the molecular orbital $\left(1 \pi_{+}\right)=1 \pi_{+}(R, \rho, z) e^{i \phi}$ with the azimuthal quantum number $M_{L}=1,{ }^{19}$

$$
\mathbf{j}_{A} 1_{\Pi_{+}}(R, \rho, z) \approx \mathbf{j}_{\left(1 \pi_{+}\right)}(R, \rho, z)=\frac{\hbar}{m_{e} \rho}\left|1 \pi_{+}(R, \rho, z)\right|^{2} \mathbf{e}_{\phi},
$$

where the cylindrical coordinates $(\rho, z, \phi)$ are used. Note that the positions of the $\mathrm{Be}$ - and O-nuclei are set to $z=0$ and $z=R$, respectively. Therefore, for $M_{L} \neq 0$ the $\phi$-component of the current density is the only one which does not vanish, and it is independent of $\phi$. Figure 6(a) shows the absolute value of the electronic current density $\left|\mathbf{j}_{\left(1 \pi_{+}\right)}\left(R_{e, X}, \rho, z\right)\right|$ at the equilibrium bond length $R=R_{e, X}$. Compared to the electronic current density of the excited state $A{ }^{1} \Pi_{+}$in $\mathrm{AlCl},{ }^{19}$ the current density of the same excited state in $\mathrm{BeO}$ is much stronger and denser. The corresponding $R$-dependent electric ring current is given as

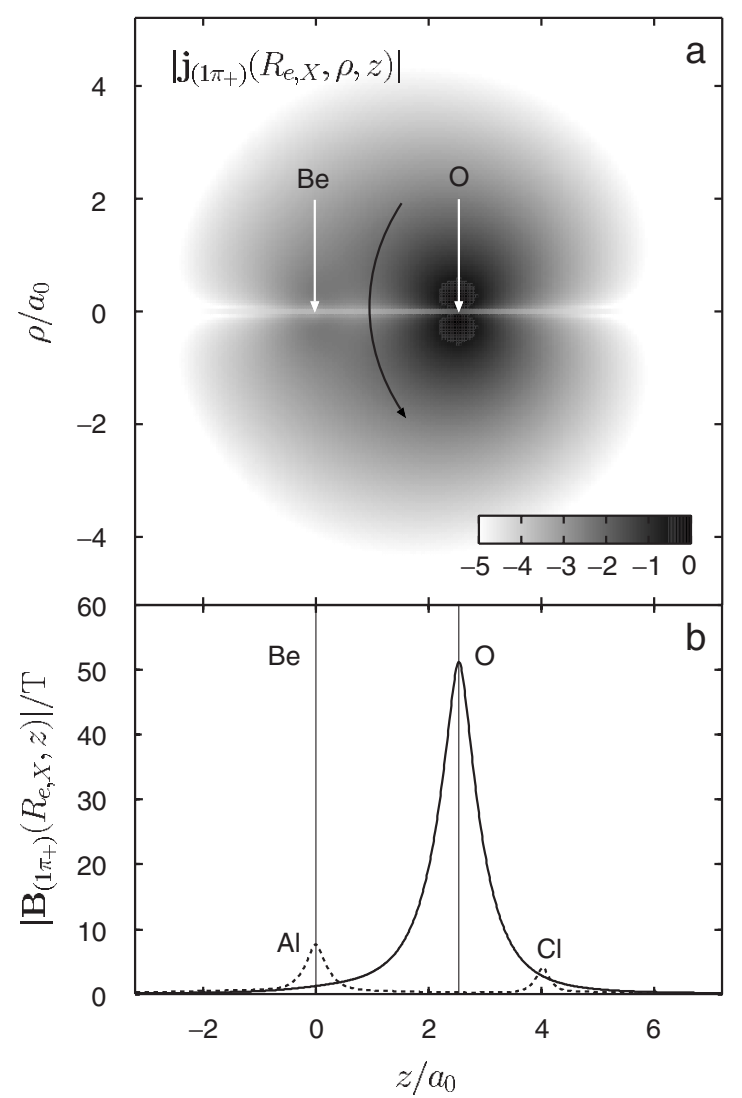

FIG. 6. Toroidal electronic current density $\left|\mathbf{j}_{\left(1 \pi_{+}\right)}\left(R_{e, X}, \rho, z\right)\right|$ [absolute values in atomic units and logarithmic scale $\left.\left(\log _{10}\right)\right]$ (panel a) and induced magnetic field along the molecular axis $\left|\mathbf{B}_{\left(1 \pi_{+}\right)}\left(R_{e, X}, z\right)\right|$ (panel b, solid curve) for the $\mathrm{A}^{1} \Pi_{+}$state of the $\mathrm{BeO}$ molecule. The dotted curve compares the magnetic field with that computed for the $A^{1} \Pi_{+}$state of the $\mathrm{AlCl}$ molecule (adapted from Ref. 19). The vertical lines indicate the positions of Be and $\mathrm{O}$ nuclei, $z=0$ and $z=R_{e, X}$, respectively.

$$
I_{A} \Pi^{1} \Pi_{+}(R) \approx I_{\left(1 \pi_{+}\right)}(R)=-\frac{e \hbar}{m_{e}} \int_{0}^{\infty} \frac{d \rho}{\rho} \int_{-\infty}^{\infty}\left|1 \pi_{+}(R, \rho, z)\right|^{2} d z
$$

At the equilibrium bond length $R=R_{e, X}$, the absolute value of $I_{\left(1 \pi_{+}\right)}\left(R_{e, X}\right)$ is $2.490 \mathrm{~mA}$ which can be compared with the corresponding value in the $\mathrm{AlCl}$ molecule $\left(I_{A}{ }^{1} \Pi_{+} \approx I_{\left(4 \pi_{+}\right)}\right.$ $=0.405 \mathrm{~mA})$ and in Mg-porphyrin $\left(I_{4}{ }^{1} E_{u+} \approx I_{\left(4 e_{g+}\right)}\right.$ $=0.085 \mathrm{~mA}) .{ }^{22}$ It corresponds to the time for the circulation of the electron in the electric ring current,

$$
T_{A}^{1} \Pi_{+}\left(R_{e, X}\right) \approx T_{\left(1 \pi_{+}\right)}\left(R_{e, X}\right)=\frac{-e}{I_{\left(1 \pi_{+}\right)}\left(R_{e, X}\right)}=64.4 \text { as, }
$$

which is short with respect to the electronic circulation time of the AlCl molecule $\left(T_{A}{ }^{1} \Pi_{+} \approx T_{\left(4 \pi_{+}\right)}=396\right.$ as $)$ and about $10^{10}$ times shorter than the lifetime of the electric ring current, which is limited by the radiative decay of the excited state, ${ }^{72} \tau_{\text {rad }, A}{ }^{1} \Pi_{+}\left(R_{e, X}\right)=1.1 \mu \mathrm{s}$. The $R$-dependent mean ring current radius is estimated as ${ }^{19}$ 


$$
\begin{aligned}
R_{A}{ }^{\mathrm{el} \Pi_{+}}(R) & \approx R_{\left(1 \pi_{+}\right)}^{\mathrm{el}}(R) \\
& =-\frac{m_{e} I_{\left(1 \pi_{+}\right)}(R)}{e \hbar \int_{0}^{\infty} \rho^{-2} d \rho \int_{-\infty}^{\infty}\left|1 \pi_{+}(R, z, \rho)\right|^{2} d z} .
\end{aligned}
$$

At $R=R_{e, X}$, the mean ring current radius is $R_{\left(1 \pi_{+}\right)}^{\mathrm{el}}\left(R_{e, X}\right)$ $=0.25 a_{0}$, which is similar to $R_{A}^{\mathrm{el}} \Pi_{+} \approx R_{\left(4 \pi_{+}\right)}^{\mathrm{el}}=0.18 a_{0}$ for $\mathrm{AlCl}$ (Ref. 19) but considerably smaller than the corresponding value in Mg-porphyrin $\left(R_{4{ }^{1} E_{u_{+}}}^{\mathrm{el}} \approx R_{\left(4 e_{g_{+}}\right)}^{\mathrm{el}}=6.32 a_{0}\right) .{ }^{22} \mathrm{In}$ atomic $n p_{ \pm}$orbitals, the mean ring current radius is $R_{\left(n p_{ \pm}\right)}^{\mathrm{el}}$ $=4 a_{0} / \pi Z_{\text {eff }}$, where $Z_{\text {eff }}$ is the effective nuclear charge. Because $R_{\left(n p_{ \pm}\right)}^{\mathrm{el}}$ is independent of the principal quantum number $n$ and since $1 / Z_{\text {eff }}$ for the $\mathrm{Al}$ and $\mathrm{O}$ nuclei are similar, the mean ring current radii for $\mathrm{AlCl}$ and $\mathrm{BeO}$ are also similar.

The electric ring current of the $A^{1} \Pi_{+}$state circulating about the molecular axis of the $\mathrm{BeO}$ molecule induces an $R$-dependence magnetic field. The induced magnetic field along the molecular $Z$-axis $(\rho=0)$ is determined using BiotSavart law as

$$
\begin{aligned}
& \mathbf{B}_{A}{ }^{1} \Pi_{+}(R, z) \approx \mathbf{B}_{\left(1 \pi_{+}\right)}(R, z) \\
& =-\frac{\mu_{0} e \hbar}{2 m_{e}} \int_{0}^{\infty} \rho^{\prime} d \rho^{\prime} \\
& \times \int_{-\infty}^{\infty} \frac{\left|1 \pi_{+}\left(R, \rho^{\prime}, z^{\prime}\right)\right|^{2}}{\left(\rho^{\prime 2}+\left(z-z^{\prime}\right)^{2}\right)^{3 / 2}} d z^{\prime} \mathbf{e}_{z} .
\end{aligned}
$$

At the equilibrium bond length $R=R_{e, X}$, the absolute value of the magnetic field at the O-nucleus $\left(z=R_{e, X}\right)$ is $52.1 \mathrm{~T}$ $=5.21 \times 10^{5} \mathrm{G}$, which is much stronger than the induced magnetic fields in $\mathrm{AlCl}\left(\left|\mathbf{B}_{A}{ }^{1} \Pi_{+}\left(z_{\mathrm{Al}}\right)\right| \approx\left|\mathbf{B}_{\left(4 \pi_{+}\right)}\left(z_{\mathrm{Al}}\right)\right|=7.70 \mathrm{~T}\right.$ and $\left.\left|\mathbf{B}_{A}{ }^{1} \Pi_{+}\left(z_{\mathrm{Cl}}\right)\right| \approx\left|\mathbf{B}_{\left(4 \pi_{+}\right)}\left(z_{\mathrm{Cl}}\right)\right|=4.13 \mathrm{~T}\right)$ and in $\mathrm{Mg}$ porphyrin $\left(\left|\mathbf{B}_{41}^{1} E_{u+}\left(z_{\mathrm{Mg}}\right)\right| \approx\left|\mathbf{B}_{\left(4 e_{g+}\right)}\left(z_{\mathrm{Mg}}\right)\right|=0.159 \mathrm{~T}\right)$. Furthermore, the corresponding absolute value of the magnetic field at the Be nucleus $(z=0)$ is $1.2 \mathrm{~T}$. The $z$-dependence of the induced magnetic field of the $\mathrm{BeO}$ molecule at $R=R_{e, X}$ is illustrated in Fig. 6(b), together with the one of the $\mathrm{AlCl}$ molecule adapted from Ref. 19 for comparison. This clearly shows that the peak height of the induced magnetic field of the $\mathrm{BeO}$ molecule is larger and its full width at half maximum is broader $\left(0.8 a_{0}\right)$ than the corresponding values of the $\mathrm{AlCl}$ molecule. There is only one peak of the induced magnetic field, i.e., at the $\mathrm{O}$ nucleus, by contrast to the one corresponding to the $\mathrm{AlCl}$ molecule, which peaks at both the $\mathrm{Al}$ and $\mathrm{Cl}$ nuclei. This confirms that the molecular $1 \pi_{+}$orbital and the corresponding electronic current density are strongly localized on the $\mathrm{O}$ nucleus, whereas the effects on the $\mathrm{Be}$ nucleus are small. Since the induced magnetic field is the result of the stationary solution of the nonrelativistic electronic Schrödinger equation for the electronic state $A{ }^{1} \Pi_{+}$, this cannot be regarded as external field. Thus, in the relativistic theory the induced magnetic field may interact with spins of inner electrons and nonzero nuclear spin of the ${ }^{17} \mathrm{O}$ nucleus $(I=5 / 2>0)$ with very small natural isotope abundance of $0.038 \%$.

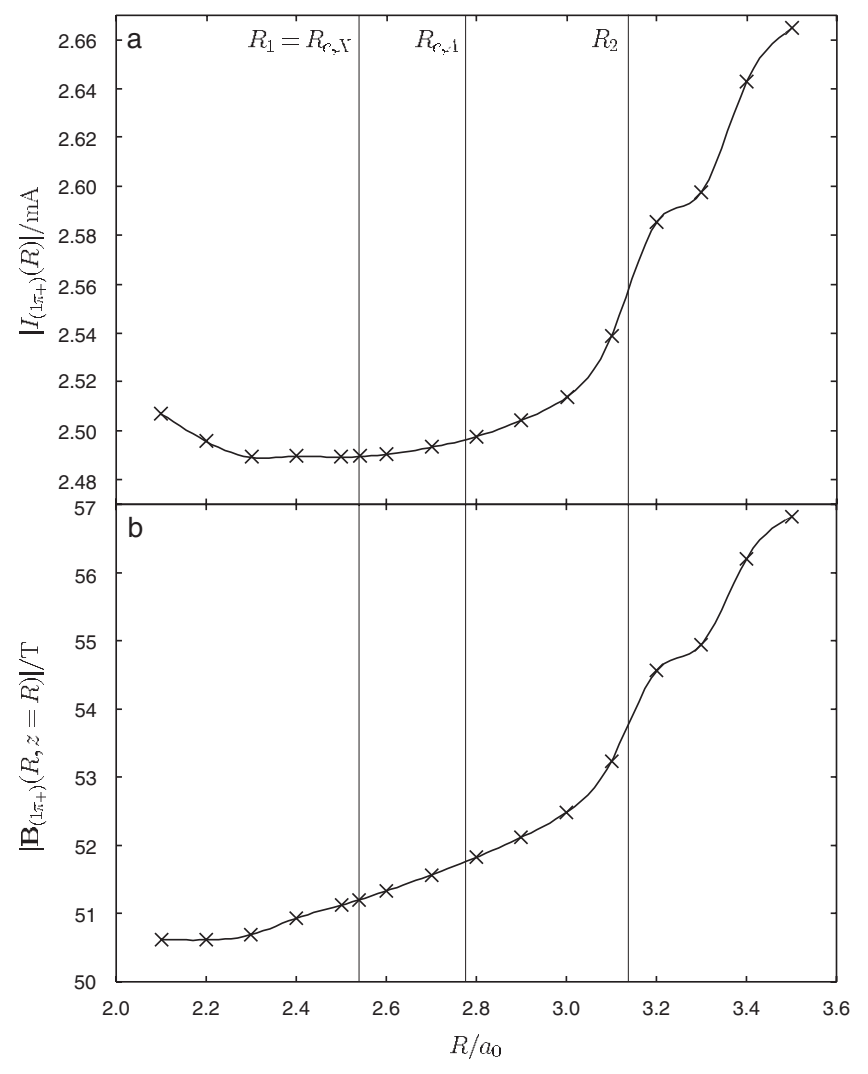

FIG. 7. Electric ring current $\left|I_{\left(1 \pi_{+}\right)}(R)\right|$ (panel a) and induced magnetic field at the $\mathrm{O}$ nucleus $\left|\mathbf{B}_{\left(1 \pi_{+}\right)}(R, z=R)\right|$ (panel b) vs the vibrational coordinate $R$ of the $\mathrm{BeO}$ molecule. The vertical lines indicate the various bond lengths $R_{1}=R_{e, X}=2.54 a_{0}, R_{e, A}=2.78 a_{0}$, and $R_{2}=3.14 a_{0}$ as shown in Fig. 2 .

The bond-length dependencies of the electric ring current [Eq. (30)] and induced magnetic field [Eq. (33)] at the O-nucleus are illustrated in Figs. 7(a) and 7(b), respectively. They show that, in general, the electric ring current and induced magnetic field increase with the bond length $R$ except at the shortest bond lengths considered. Nevertheless, the corresponding slopes are relatively small, in particular, between the classical turning points $R_{1}=R_{e, X}$ and $R_{2}$, where the electric ring current is constant to within $2.7 \%$ and the induced magnetic field to within $5.0 \%$. This implies that the variations in the electric ring current and induced magnetic field in the course of molecular vibrations in the excited state are negligible.

In the $R \rightarrow \infty$ limit, the $\mathrm{BeO}$ molecule in the excited $A{ }^{1} \Pi_{+}$state dissociates to $\mathrm{Be}\left(1{ }^{1} S\right)+\mathrm{O}\left(1{ }^{1} D_{+1}\right)$. In this limit the electric ring current about the Be nucleus vanishes because the ground state $1{ }^{1} S$ of the Be atom has zero azimuthal quantum number $\left(M_{L}=0\right)$. By contrast, strong electric ring current persists about the $\mathrm{O}$-nucleus, since the lowest singlet state $1{ }^{1} D_{M_{L}}$ of the O-atom is fivefold degenerate $\left(M_{L}=0, \pm 1, \pm 2\right)$ and the $M_{L} \neq 0$ components carry nonzero electric ring current. The singlet state $1{ }^{1} D_{+1}$ corresponds to the dominant electronic configuration $\cdots\left(2 p_{-}\right)$ $\times\left(2 p_{0}\right)\left(2 p_{+}\right)^{2}$ with dominant net ring current of the atomic $2 p_{+}$orbital, which, as noted above, is strong. The corresponding values for the $\mathrm{O}$ atom are $\left|I_{1}{ }^{1} D_{+1}\right| \approx\left|I_{\left(2 p_{+}\right.}\right|$ $=2.85 \mathrm{~mA}, T_{1}{ }^{1} D_{+1} \approx T_{\left(2 p_{+}\right)}=56.2 \mathrm{as}, R_{1 D_{+1}}^{\mathrm{el}} \approx R_{\left(2 p_{+}\right)}^{\mathrm{el}}=0.24 a_{0}$, and $\left|\mathbf{B}_{1}{ }^{1} D_{+1}\right| \approx\left|\mathbf{B}_{\left(2 p_{+}\right)}\right|=62.0 \mathrm{~T}$ at the O-nucleus. Thus, the 
effects for the O-atom are somewhat stronger than for the $\mathrm{BeO}$ molecule because in the dissociation limit the responsible orbital $\left(1 \pi_{+}\right) \rightarrow\left(2 p_{+}\right)$is fully localized on the $\mathrm{O}$ atom.

Until now, it was assumed that the final state is stationary, e.g., the $A^{1} \Pi_{+}$state, which can be excited from the electronic ground state $X^{1} \Sigma^{+}$by an optimized right circularly polarized laser pulse. In the case that the final state is not stationary, for example, a superposition of the $X^{1} \Sigma^{+}$and $A^{1} \Pi_{+}$states, which can be excited by means of a right circularly polarized $\pi / 2$ laser pulse, ${ }^{21,23,24}$ then the resulting electronic density, electronic current density, and induced magnetic field are no longer stationary. The time-dependent electronic current density after the end of the laser pulse $\left(t \geq t_{f}\right)$ is derived from Eq. (24) and Eq. (19) of Ref. 19,

$$
\mathbf{j}(R, \rho, z, \phi, t)=\sum_{i=0}^{i_{\max }} P_{i}\left(t_{f}\right) \mathbf{j}_{i}(R, \rho, z)+\mathbf{j}_{\text {int }}(R, \rho, z, \phi, t),
$$

where $\mathbf{j}_{i}(R, \rho, z)$ is the electronic current density of the state $\left|\Psi_{i}(R)\right\rangle$ and is independent of time $t$ and of the azimuthal angle $\phi$, in contrast to the interference term $\mathbf{j}_{\text {int }}(R, \rho, z, \phi, t)$. Time averaging equation (34) and using $\mathbf{j} 1_{\Sigma}(R, \rho, z)=0$, $\mathbf{j}^{1} \Pi_{-}(R, \rho, z)=-\mathbf{j}{ }^{1} \Pi_{+}(R, \rho, z)$ and $\mathbf{j}^{1} \Delta_{\Delta_{-}}(R, \rho, z)=-\mathbf{j}{ }^{1} \Delta_{+}(R, \rho, z)$, we have

$$
\begin{aligned}
& \overline{\mathbf{j}}(R, \rho, z)=\left(P_{A}{ }^{1} \Pi_{+}\left(t_{f}\right)-P_{A}{ }^{1} \Pi_{-}\left(t_{f}\right)\right) \mathbf{j}_{A}{ }^{1} \Pi_{+} \\
&+(R, \rho, z) \\
&+\left(P_{C}{ }^{1} \Delta_{+}\right. \\
&
\end{aligned}
$$

which is independent of the azimuthal angle $\phi$. In the present work, $P_{A}{ }^{1} \Pi_{+}\left(t_{f}\right) \approx 1$ and other populations are negligible, thus $\mathbf{j}(R, \rho, z, \phi, t) \approx \overline{\mathbf{j}}(R, \rho, z) \approx \mathbf{j}_{A}{ }^{1} \Pi_{+}(R, \rho, z)$, cf. Eq. (29). For example, the time-averaged electronic current density of a superposition state consisting of $X^{1} \Sigma^{+}$and $A^{1} \Pi_{+}$states is $\overline{\mathbf{j}}(R, \rho, z)=P_{A}{ }^{1} \Pi_{+}\left(t_{f}\right) \mathbf{j}_{A}{ }^{1} \Pi_{+}(R, \rho, z)$, i.e., it is proportional to the final population $P_{A}{ }^{1} \Pi_{+}\left(t_{f}\right)$ of the $A{ }^{1} \Pi_{+}$state. With Eqs. (20) and (22) of Ref. 19, the corresponding time-averaged electric ring current and induced magnetic field along the molecular axis take the form

$$
\begin{aligned}
\bar{I}(R)= & \left(P_{A}{ }^{1} \Pi_{+}\left(t_{f}\right)-P_{A}{ }^{1} \Pi_{-}\left(t_{f}\right)\right) I_{A}{ }^{1} \Pi_{+} \\
& +\left(P_{C}{ }^{1} \Delta_{+}\right. \\
& \left(t_{f}\right)-P_{C}{ }^{1} \Delta_{-}
\end{aligned}
$$

and

$$
\begin{aligned}
& \overline{\mathbf{B}}(R, z)=\left(P_{A}{ }^{1} \Pi_{+}\left(t_{f}\right)-P_{A}{ }^{1} \Pi_{-}\left(t_{f}\right)\right) \mathbf{B}_{A}{ }^{1} \Pi_{+}(R, z) \\
& +\left(P_{C^{1} \Delta_{+}}\left(t_{f}\right)-P_{C^{1} \Delta_{-}}\left(t_{f}\right)\right) \mathbf{B}_{C{ }^{1} \Delta_{+}}(R, z)+\cdots,
\end{aligned}
$$

respectively. Again, in the present work, $\bar{I}(R) \approx I_{A}{ }^{1} \Pi_{+}(R)$ and $\overline{\mathbf{B}}(R, z) \approx \mathbf{B}_{A}{ }^{1} \Pi_{+}(R, z)$, cf. Eqs. (30) and (33), respectively.

The results presented above, as well as the results of previous publications on the same topic, assumed perfect orientation. In reality, as discussed in the previous section, laser induced orientation can be very sharp but is never perfect, e.g., for $\mathrm{BeO}$ at $T=1 \mathrm{~K}\left|\langle\cos \theta\rangle_{T}\left(t^{*}\right)\right|=0.85 \neq 1$, see Sec. II and Fig. 3. Application of the same right circularly polarized laser pulse as in the calculation leading to Figs. 3(a) and 3(b) to the nonperfectly oriented molecule gives rise to additional dipole-allowed transitions. For example, the transition $X^{1} \Sigma^{+} \leftrightarrow B{ }^{1} \Sigma^{+}$becomes allowed because the projection of the molecular $Z$-axis on the laboratory $x$ - or $y$-axis is nonzero (while small). The final population of the $A{ }^{1} \Pi_{+}$state is thus smaller and the ones of the $X^{1} \Sigma^{+}, A^{1} \Pi_{-}$, and $B^{1} \Sigma^{+}$states larger in the case of imperfect than in the case of perfect orientation, resulting in a smaller $P_{A}{ }^{1} \Pi_{+}\left(t_{f}\right)-P_{A}{ }^{1} \Pi_{-}\left(t_{f}\right)$ difference. Thus, the electric ring current about the molecular $Z$-axis and the induced magnetic field along the $Z$-axis are expected to be smaller for imperfect orientation than in the results provided above, cf. Eqs. (36) and (37). In the case of perpendicular orientation, the final populations of the $A^{1} \Pi_{+}$ and $A^{1} \Pi_{-}$states are equal, and there are no electric ring current about the molecular axis, cf. Eq. (36). An electric ring current about the axis perpendicular to the molecular axis will arise in the case of nonzero population of the $B^{1} \Sigma^{+}$ state, but this ring current is not stationary and toroidal. Because the projection of the molecular $Z$-axis onto the laboratory $x$ and $y$ axes remains small, and since the excitation frequency of the $B{ }^{1} \Sigma^{+}$state at $R=R_{e, X}$ is twice as large as the laser frequency, the final population of the $B^{1} \Sigma^{+}$state will be small. Thus, deviations from the idealized limit of perfect orientation are expected to modify the detail but not the qualitative physics predicted.

Before concluding this section, it is pertinent to briefly comment on the feasibility of an experimental demonstration of toroidal current in oriented $\mathrm{BeO}$. The electronic transitions between the $X^{1} \Sigma^{+}, A^{1} \Pi$, and $B^{1} \Sigma^{+}$states of the BeO molecule in the gas phase have been observed. ${ }^{73}$ The corresponding experimental spectroscopic constants of these three states are listed in Refs. 57 and 74. Several documented methods could be applied to produce gas phase $\mathrm{BeO}$ molecules. These include the vaporization approach of Ref. 75 or that of Refs. 76 and 77, the laser ablation method of Refs. 78-80, and the sputtering method of Ref. 79 and that of Ref. 81. We remark that the rate constant of the oxidation, $\mathrm{Be}+\mathrm{O}_{2} \rightarrow \mathrm{BeO}+\mathrm{O}$, has been computed in the 770-1200 K temperature. ${ }^{82}$ Furthermore, Be oxides in the gas phase are dominated by the polymeric form $(\mathrm{BeO})_{n}(n>1)$ because of the high boiling point of $\mathrm{BeO}$. The possibility of extracting $\mathrm{BeO}$ from the polymeric states is pointed out in Ref. 76. Laser orientation by means of HCPs has been discussed in several recent articles. ${ }^{61,63}$ (The generation of high-power sub-single-cycle pulses is discussed, e.g., in Ref. 83.) Finally, the detection of the electric ring current could be accomplished by high harmonic generation by means of elliptically polarized laser pulses. $^{33}$

\section{CONCLUSIONS}

In this work we illustrated sharp, field-free (postpulse) orientation of the $\mathrm{BeO}$ molecule (Sec. III) by means of an optimized, linearly polarized laser pulse of short duration with respect to the rotational period. Excitation of the oriented molecule by means of an optimized right circularly polarized laser pulse in resonance with the first excited $A^{1} \Pi_{+}$state was shown to give rise to strong unidirectional 
electric ring current about the molecular axis and a corresponding substantial induced magnetic field at the oxygen nucleus (Sec. IV). Specifically, we found a ring current of magnitude of $2.49 \mathrm{~mA}$ and a magnetic field of $51.2 \mathrm{~T}$ at the equilibrium configuration, which depend weakly on the internuclear distance, converging to $2.85 \mathrm{~mA}$ and $62.0 \mathrm{~T}$ in the dissociation limit. Our results were based on CASSCF/ CASPT2 calculations of the potential curves and dipole matrix elements of the $\mathrm{BeO}$ molecule, ${ }^{4,49}$ time-dependent quantum mechanical calculation of the alignment dynamics, ${ }^{37}$ and quantum simulations of the ring current and magnetic field. ${ }^{19}$

Our considerations in choosing the $\mathrm{BeO}$ molecule in an effort to identify a molecule that should exhibit strong effects were detailed in Sec. II, along with a list of the criteria that are required for observation of ring currents in a general system. We remark, however, that the effects predicted here are rather general. Strong electric ring currents and induced magnetic fields can in principle be observed in atoms, ions, linear molecules or molecular ions, and in states other than the lowest excited degenerate singlet states that were considered so far. For example, the excited bound state $C{ }^{1} \Delta_{+}$ $\left(M_{L}=2\right)$ of the $\mathrm{BeO}$ molecule, see Fig. 2, with corresponding dominant electronic configuration $\cdots\left(1 \pi_{+}\right)^{2}\left(1 \pi_{-}\right)\left(2 \pi_{+}\right)$ (weight of $95.7 \%$ ), has two separate unidirectional electronic ring currents of molecular $1 \pi_{+}$and $2 \pi_{+}$orbitals, hence the corresponding electric ring current and induced magnetic field are expected to be stronger than for the $A{ }^{1} \Pi_{+}$explored above. This excited $C^{1} \Delta_{+}$state has a small potential barrier at about $R=4.5 a_{0}$ due to an avoided crossing ${ }^{43}$ which leads to dissociation of the $\mathrm{BeO}$ molecule to $\mathrm{Be}\left(1^{1} S\right)+\mathrm{O}\left(1^{1} D_{+2}\right)$. The singlet $\mathrm{O}$-atom $1{ }^{1} D_{+2}$ state (with $M_{L}=2$ ) corresponds to the dominant electronic configuration $\cdots\left(2 p_{0}\right)^{2}\left(2 p_{+}\right)^{2}$ and carries an electric ring current of $5.70 \mathrm{~mA}$ and an induced magnetic field of 124.0 T. These values are twice as large as the corresponding values for the singlet state $1{ }^{1} D_{+1}$ $\left(M_{L}=1\right)$ of the O-atom because two (rather than a single) electrons with opposite spins and the same spatial atomic $2 p_{+}$ orbital circulate with the same electronic circulation time and mean ring current radius about the $\mathrm{O}$ nucleus. [Although the effects considered are atomic, rather than molecular, in practice it is simpler to produce the lowest singlet state $1{ }^{1} D_{M_{L}}$ of the O-atom with a desired nonzero azimuthal quantum number $\left(M_{L}= \pm 1, \pm 2\right)$ through dissociation of an appropriate molecular state than directly from the ground (triplet) state $1^{3} \mathrm{P}$ of the O-atom.]

Another example is the LiF molecule, which has a dissociative excited $A^{1} \Pi_{+}$state that can be excited by an optimized right circularly polarized laser pulse from the ground state $X^{1} \Sigma^{+}$, using the method described in Sec. IV. Upon excitation to the $A{ }^{1} \Pi_{+}$state, the molecule dissociates into $\mathrm{Li}\left(1{ }^{2} S\right)+\mathrm{F}\left(1^{2} P_{+}\right)$. Regardless of the spin effects, the ground $1{ }^{2} S$ state of the Li-atom carries no electric ring current, but the ground $1{ }^{2} P_{+}\left(M_{L}=1\right)$ state of the F-atom, corresponding to the dominant electronic configuration $\cdots\left(2 p_{-}\right)\left(2 p_{0}\right)^{2}\left(2 p_{+}\right)^{2}$, carries electric ring current of magnitude of $3.80 \mathrm{~mA}$ and an induced magnetic field of $95.2 \mathrm{~T}$ at the F-nucleus. The corresponding electronic circulation time is 42.2 as and the mean ring current radius is $0.21 a_{0}$. Thus, the effects for the F-atom are stronger than the ones for the
O-atom because of the large effective nuclear charge $Z_{\text {eff }}$ of the $\mathrm{F}$ atom. Finally, as discussed in Ref. 18, the electric ring currents and induced magnetic fields of some electronic states of atomic and molecular ions can be stronger than those of the corresponding neutral systems, an example is the lowest excited singlet states $1{ }^{1} D_{M_{L}}\left(M_{L}= \pm 1, \pm 2\right)$ of the $\mathrm{F}^{+}$ion whose $Z_{\text {eff }}$ is larger than that of the F-atom.

Before concluding, it is important to comment on the long time dynamics that were excluded from the discussion of the previous sections. Previous work has predicted that electric ring currents would persist for nano- and even microseconds in stable excited states. In practice, however, the ring currents rely on sharp orientation of the molecule with respect to the excitation field, and the only way of producing well-oriented systems free of external fields at present is the nonadiabatic alignment/orientation method. This approach provides (in linear or symmetric top molecules) a series of transient alignment (orientation) peaks whose duration is shorter the better aligned (oriented) the molecule and whose separation is determined by the molecular rotational period. $^{36,37}$ Thus, the ring current would persist for a short period (see Fig. 3) but will recur periodically in time, following the regular revival pattern of stable bound state rigid rotors. At longer times, of the order of the inverse of the rotation-vibration coupling energy, the assumption of separability of the rotational and vibrational motions is no longer valid and the rotational revival structure gradually dephases, see Ref. 59. For linear molecules, the only source of rotationvibration coupling is centrifugal effects, which are typically weak, appearing on 0.1-1 ns time scales. Provided that coherence is maintained, the rovibrational levels rephase at later times and the purely rotational, rigid-rotor-like pattern reappears, forming a longer time rovibrational revival pattern that is superimposed on the standard rigid-rotor revival pattern. It would be interesting for future work to observe these effects on the electric ring currents induced in linear molecules.

\section{ACKNOWLEDGMENTS}

We thank Professor J. Manz and Dr. N. Elghobashi for stimulating discussions and Professor T. Baumert and Dr. T. Siebert for experimental advice. T.S. is grateful to the Alexander von Humboldt Foundation for a generous award and for support of a visit to Berlin that enabled this research. Support of the Deutsche Forschungsgemeinschaft (Project Nos. Ma 515/23-1 and subsequently Sfb 450/TPC1), the Agentur für Arbeit Berlin-Nord, the Spanish MEC (Project Nos. CTQ2007-61260 and CSD2007-0010 ConsoliderIngenio in Molecular Nanoscience), and the U.S. Department of Energy (Grant No. DAAD19-03-R0017) is gratefully acknowledged.

\footnotetext{
${ }^{1}$ M. Drescher, M. Hentschel, R. Kienberger, G. Tempea, C. Spielmann, G. A. Reider, P. B. Corkum, and F. Krausz, Science 291, 1923 (2001).

${ }^{2}$ P. Agostini and L. F. DiMauro, Rep. Prog. Phys. 67, 813 (2004).

${ }^{3}$ A. Scrinzi, M. Y. Ivanov, R. Kienberger, and D. M. Villeneuve, J. Phys. B 39, R1 (2006).

${ }^{4}$ T. Kato and H. Kono, Chem. Phys. Lett. 392, 533 (2004).

${ }^{5}$ J. Caillat, J. Zanghellini, M. Kitzler, O. Koch, W. Kreuzer, and A. Scrinzi, Phys. Rev. A 71, 012712 (2005).
} 
${ }^{6}$ A. I. Kuleff, J. Breidbach, and L. S. Cederbaum, J. Chem. Phys. 123, 044111 (2005).

${ }^{7}$ P. Krause, T. Klamroth, and P. Saalfrank, J. Chem. Phys. 123, 074105 (2005).

${ }^{8}$ M. Awasthi and A. Saenz, J. Phys. B 39, S389 (2006).

${ }^{9}$ S. Chelkowski, G. L. Yudin, and A. D. Bandrauk, J. Phys. B 39, S409 (2006).

${ }^{10}$ S. Baier, C. Ruiz, L. Plaja, and A. Becker, Phys. Rev. A 74, 033405 (2006).

${ }^{11}$ T. K. Kjeldsen, L. B. Madsen, and J. P. Hansen, Phys. Rev. A 74, 035402 (2006).

${ }^{12}$ R. Fischer, M. Lein, and C. H. Keitel, Phys. Rev. Lett. 97, 143901 (2006).

${ }^{13}$ I. Barth, J. Manz, and G. K. Paramonov, Mol. Phys. 106, 467 (2008).

${ }^{14}$ G. K. Paramonov, Chem. Phys. Lett. 411, 350 (2005).

${ }^{15}$ H. Kono, Y. Sato, M. Kanno, K. Nakai, and T. Kato, Bull. Chem. Soc. Jpn. 79, 196 (2006)

${ }^{16}$ A. D. Bandrauk, S. Chelkowski, and G. L. Yudin, in Photon-based Nanoscience and Nanobiotechnology, edited by J. J. Dubowski and S. Tanev (Springer, Dordrecht, 2006), p. 31.

${ }^{17}$ A. Palacios, H. Bachau, and F. Martín, Phys. Rev. A 75, 013408 (2007)

${ }^{18}$ I. Barth and J. Manz, Phys. Rev. A 75, 012510 (2007).

${ }^{19}$ I. Barth, J. Manz, and L. Serrano-Andrés, Chem. Phys. 347, 263 (2008).

${ }^{20}$ M. Nest, F. Remacle, and R. D. Levine, New J. Phys. 10, 025019 (2008)

${ }^{21}$ I. Barth and J. Manz, Angew. Chem. 118, 3028 (2006); Angew. Chem., Int. Ed. 45, 2962 (2006).

${ }^{22}$ I. Barth, J. Manz, Y. Shigeta, and K. Yagi, J. Am. Chem. Soc. 128, 7043 (2006)

${ }^{23}$ I. Barth and J. Manz, in Femtochemistry VII: Fundamental Ultrafast Processes in Chemistry, Physics, and Biology, edited by A. W. Castleman, Jr. and M. L. Kimble (Elsevier, Amsterdam, 2006), p. 441.

${ }^{24}$ I. Barth, L. González, C. Lasser, J. Manz, and T. Rozgonyi, in Coherent Control of Molecules, edited by B. Lasorne and G. A. Worth (CCP6, Daresbury, 2006), p. 18.

${ }^{25}$ K. Nobusada and K. Yabana, Phys. Rev. A 75, 032518 (2007).

${ }^{26}$ A. Matos-Abiague and J. Berakdar, Phys. Rev. Lett. 94, 166801 (2005).

${ }^{27}$ Y. V. Pershin and C. Piermarocchi, Phys. Rev. B 72, 245331 (2005).

${ }^{28}$ E. Räsänen, A. Castro, J. Werschnik, A. Rubio, and E. K. U. Gross, Phys. Rev. Lett. 98, 157404 (2007).

${ }^{29}$ M. Kanno, H. Kono, and Y. Fujimura, Angew. Chem. 118, 8163 (2006); Angew. Chem., Int. Ed. 45, 7995 (2006).

${ }^{30}$ M. Kanno, K. Hoki, H. Kono, and Y. Fujimura, J. Chem. Phys. 127, 204314 (2007).

${ }^{31}$ I. Barth, J. Manz, and P. Sebald, Chem. Phys. 346, 89 (2008).

${ }^{32}$ I. Barth, J. Manz, G. Pérez-Hernández, and P. Sebald, Z. Phys. Chem. 222, 1311 (2008)

${ }^{33}$ X. Xie, A. Scrinzi, M. Wickenhauser, A. Baltuška, I. Barth, and M. Kitzler, Phys. Rev. Lett. 101, 033901 (2008).

${ }^{34}$ J. Itatani, J. Levesque, D. Zeidler, H. Niikura, H. Pépin, J. C. Kieffer, P. B. Corkum, and D. M. Villeneuve, Nature (London) 432, 867 (2004).

${ }^{35}$ T. Seideman, Annu. Rev. Phys. Chem. 53, 41 (2002).

${ }^{36}$ H. Stapelfeldt and T. Seideman, Rev. Mod. Phys. 75, 543 (2003).

${ }^{37}$ T. Seideman and E. Hamilton, Adv. At., Mol., Opt. Phys. 52, 289 (2006).

${ }^{38}$ M. Yoshimine, J. Chem. Phys. 40, 2970 (1964).

${ }^{39}$ H. F. Schaefer III, J. Chem. Phys. 55, 176 (1971).

${ }^{40}$ G. H. F. Diercksen, A. J. Sadlej, and M. Urban, Chem. Phys. 158, 19 (1991)

${ }^{41}$ J. D. Watts, M. Urban, and R. J. Bartlett, Theor. Chim. Acta 90, 341 (1995).

${ }^{42}$ W. M. Huo, K. F. Freed, and W. Klemperer, J. Chem. Phys. 46, 3556 (1967).

43 R. J. Buenker, H.-P. Liebermann, L. Pichl, M. Tachikawa, and M. Kimura, J. Chem. Phys. 126, 104305 (2007).

${ }^{44}$ H. Ghalila, S. Lahmar, Z. B. Lakhdar, and M. Hochlaf, J. Phys. B 41, 205101 (2008);

${ }^{45}$ K. Andersson, P.-Å. Malmqvist, and B. O. Roos, J. Chem. Phys. 96,
1218 (1992)

${ }^{46}$ C. Zener, Phys. Rev. 36, 51 (1930).

${ }^{47}$ J. C. Slater, Phys. Rev. 36, 57 (1930)

${ }^{48}$ L. Serrano-Andrés, M. Merchán, I. Nebot-Gil, R. Lindh, and B. O. Roos, J. Chem. Phys. 98, 3151 (1993).

${ }^{49}$ B. O. Roos, K. Andersson, M. P. Fülscher, P.-A. Malmqvist, L. SerranoAndrés, K. Pierloot, and M. Merchán, Adv. Chem. Phys. 93, 219 (1996).

${ }^{50}$ L. Serrano-Andrés, N. Forsberg, and P.-A. Malmqvist, J. Chem. Phys. 108, 7202 (1998).

${ }^{51}$ A. C. Borin and L. Serrano-Andrés, Chem. Phys. 262, 253 (2000).

${ }^{52}$ L. Serrano-Andrés, M. Merchán, and A. C. Borin, Chem.-Eur. J. 12, 6559 (2006).

${ }^{53}$ B. O. Roos and P.-Å. Malmqvist, Phys. Chem. Chem. Phys. 6, 2919 (2004).

${ }^{54}$ N. Forsberg and P.- ̊. Malmqvist, Chem. Phys. Lett. 274, 196 (1997).

${ }^{55}$ G. Karlström, R. Lindh, P.-Å. Malmqvist, B. O. Roos, U. Ryde, V. Veryazov, P.-O. Widmark, M. Cossi, B. Schimmelpfennig, P. Neogrady, and L. Seijo, Comput. Mater. Sci. 28, 222 (2003).

${ }^{56}$ V. Veryazov, P.-O. Widmark, L. Serrano-Andrés, R. Lindh, and B. O. Roos, Int. J. Quantum Chem. 100, 626 (2004).

${ }^{57}$ K. P. Huber and G. Herzberg, Molecular Spectra and Molecular Structure (Van Nostrand Reinhold, New York, 1979).

${ }^{58}$ L. P. Theard and D. L. Hildenbrand, J. Chem. Phys. 41, 3416 (1964).

${ }^{59}$ S. C. Althorpe and T. Seideman, J. Chem. Phys. 113, 7901 (2000).

${ }^{60}$ C. M. Dion, A. Keller, and O. Atabek, Eur. Phys. J. D 14, 249 (2001).

${ }^{61}$ M. Machholm and N. E. Henriksen, Phys. Rev. Lett. 87, 193001 (2001).

${ }^{62}$ A. Ben Haj-Yedder, A. Yuger, C. M. Dion, E. Cancés, A. Keller, C. Le Bris, and O. Atabek, Phys. Rev. A 66, 063401 (2002).

${ }^{63}$ N. Elghobashi-Meinhardt, L. González, I. Barth, and T. Seideman, "Fewcycle laser pulses to obtain spatial separation of $\mathrm{OHF}^{-}$dissociation products," J. Chem. Phys. (submitted).

${ }^{64}$ J. Rauch and G. Mourou, Proc. Am. Math. Soc. 134, 851 (2006).

${ }^{65}$ S. Chelkowski and A. D. Bandrauk, Phys. Rev. A 71, 053815 (2005).

${ }^{66}$ B. Fischer, M. Hoffmann, H. Helm, G. Modjesch, and P. U. Jepsen, Semicond. Sci. Technol. 20, S246 (2005).

${ }^{67}$ A. R. Edmonds, Angular Momentum in Quantum Mechanics (Princeton University Press, Princeton, 1957).

${ }^{68}$ R. N. Zare, Angular Momentum (Wiley, New York, 1988).

${ }^{69}$ P. Deuflhard and F. Bornemann, Numerische Mathematik II: Gewöhnliche Differentialgleichungen (de Gruyter, Berlin, 2002).

${ }^{70}$ T. Seideman, Phys. Rev. Lett. 83, 4971 (1999).

${ }^{71}$ K. Mishima, M. Hayashi, J. Yi, S. H. Lin, H. L. Selzle, and E. W. Schlag, Phys. Rev. A 66, 033401 (2002)

${ }^{72}$ A. Hinchliffe and R. W. Munn, Molecular Electromagnetism (Wiley, Chichester, 1985).

${ }^{73}$ A. Lagerqvist, Ph.D. Thesis, University of Stockholm, 1948.

${ }^{74}$ H. Lavendy, B. Pouilly, and J. M. Robbe, J. Mol. Spectrosc. 103, 379 (1984).

${ }^{75}$ W. A. Chupka, J. Berkowitz, and C. F. Giese, J. Chem. Phys. 30, 827 (1959).

${ }^{76}$ R. S. C. Mcintyre, D. C. Grégoire, and C. L. Chakrabarti, J. Anal. At. Spectrom. 12, 547 (1997).

${ }^{77}$ V. Majidi, N. Xu, and R. G. Smith, Spectrochim. Acta, Part B 55, 3 (2000).

${ }^{78}$ P. Milani and W. A. deHeer, Rev. Sci. Instrum. 61, 1835 (1990).

${ }^{79}$ F. L. King, B. I. Dunlap, and D. C. Parent, J. Chem. Phys. 94, 2578 (1991)

${ }^{80}$ M. Foltin, G. J. Stueber, and E. R. Bernstein, J. Chem. Phys. 111, 9577 (1999).

${ }^{81}$ P.-G. Fournier, A. Nourtier, V. I. Shulga, and M. Ait El Fqih, Nucl. Instrum. Methods Phys. Res. B 230, 577 (2005).

${ }^{82}$ O. E. Kashireninov, V. A. Kuznetsov, and G. B. Manelis, AIAA J. 15, 1035 (1977)

${ }^{83}$ D. You, R. R. Jones, P. H. Bucksbaum, and D. R. Dykaar, Opt. Lett. 18, 290 (1993). 